\title{
Optical Demultiplexing Based on Four-Wave Mixing in Semiconductor Optical Amplifiers
}

\author{
Narottam Das ${ }^{1,2}$ and Hitoshi Kawaguchi ${ }^{3}$ \\ ${ }^{1}$ Department of Electrical and Computer Engineering, Curtin University, \\ ${ }^{2}$ School of Engineering, Edith Cowan University, \\ ${ }^{3}$ Graduate School of Materials Science, Nara Institute of Science and Technology, \\ ${ }_{1,2}$ Australia \\ 3 Japan
}

\section{Introduction}

Four-wave mixing (FWM) in semiconductor optical amplifiers (SOAs) has several important features, such as, high speed and high FWM conversion efficiency as well as optical demultiplexing (DEMUX) (Mecozzi et al., 1995; Mecozzi \& Mфrk, 1997; Das et al., 2000). The are several applications of FWM in SOAs for all-optical devices, such as, wavelength converters (Vahala et al., 1996), optical samplers (Inoue \& Kawaguchi, 1998), optical phase conjugators (Kikuchi \& Matsumura, 1998) and optical multiplexers/demultiplexers (Kawanishi et al., 1997; Kawanishi et al., 1994; Morioka et al., 1996; Uchiyama et al., 1998; Tomkos et al., 1999; Kirita et al., 1998; Buxens et al., 2000) have been demonstrated for optical communication systems. When a pulse of a time-multiplexed signal train (for example, a probe pulse) and a pump pulse are injected simultaneously into an SOA, gain and refractive index in the SOA are modulated and an FWM signal is generated by the modulations. Thus, we can obtain a demultiplexed signal as an FWM signal at the output of SOA. All-optical demultiplexing has been experimentally demonstrated up to $200 \mathrm{Gbit} / \mathrm{s}$ (Morioka et al., 1996). Tomkos et al., (Tomkos et al., 1999) suggested a number of ways to improve the performance of the dual-pump demultiplexer at $40 \mathrm{Gbit} / \mathrm{s}$ as follows; adjustment of the input wavelengths at the peak gain wavelength of the SOA under saturation conditions, the use of higher pump power at the input of the device, or/and the use of pulsed pumps with short pulsewidths. For the higher bit-rate, the overlap of the input to the FWM signal pulses may appear both in the time and spectral domain. The pattern effect may also appear in the FWM signal due to the slow components of the optical nonlinearities in SOAs (Saleh \& Habbab, 1990). These effects degrade the usefulness of the FWM in SOAs as a practical DEMUX device in optical network/ communication systems. Therefore, it is very important to analysis the optical DEMUX characteristics based on FWM in SOAs for the ultrafast multi-bit input optical pulses.

The analyses of FWM in SOAs between short optical pulses have been widely reported (Shtaif \& Eisenstein, 1995; Shtaif et al., 1995; Xie et al., 1999; Tang \& Shore, 1998; Tang \& Shore, 1999a; Tang \& Shore, 1999b; Das et al., 2000). The FWM conversion efficiency (Shtaif et al., 1995; Xie et al., 1999; Tang \& Shore, 1998; Tang \& Shore, 1999a; Mork \& Mecozzi, 1997) 
the chirp of mixing pulses (Tang \& Shore, 1999b; Das et al., 2000), and the pump-probe time delay dependency of the FWM conversion efficiency (Shtaif \& Eisenstein, 1995; Shtaif et al., 1995; Mecozzi \& Mфrk, 1997; Das et al., 2007; Das et al., 2011) have been reported. On the contrary, however, there are only a few reports on analyses of FWM in SOAs used for demultiplexing time-division multiplexed data streams at ultra-high bit rates. Eiselt (Eiselt, 1995) reported the optimum control pulse energy and width with respect to the switching efficiency, channel crosstalk, and jitter tolerance. In those calculations, a very simple model of time-resolved gain saturation was used, which only took into account the gain recovery time. The FWM model was also very simple, in which the optical output power of the converted signal was proportional to the product of the squared pump output power and signal output power. Shtaif and Eisenstein (Shtaif \& Eisenstein, 1996) calculated the error probabilities for time-domain DEMUX. Therefore, a detail and accurate analysis is required in order to clarify the performance of optical DEMUX based on FWM in SOAs for highspeed optical communication systems.

In this Chapter, we present detail numerical modeling/simulation results of FWM characteristics for the solitary probe pulse and optical DEMUX characteristics for multi-bit (multi-probe and/or pump) pulses in SOAs by using the finite-difference beam propagation method (FD-BPM) (Das et al., 2000; Razaghi et al., 2009). These simulations are based on the nonlinear propagation equation considering the group velocity dispersion, self-phase modulation (SPM), and two-photon absorption (TPA), with the dependencies on the carrier depletion $(\mathrm{CD})$, carrier heating $(\mathrm{CH})$, spectral-hole burning $(\mathrm{SHB})$, and their dispersions, including the recovery times in SOAs (Hong et al., 1996). For the simulation of solitary probe pulse, we obtain an optimum input pump pulsewidth from a viewpoint of ON/OFF ratios. For the simulation of optical DEMUX characteristics, we evaluate the ON/OFF ratios and the pattern effect of FWM signals for the multi-probe pulses. We have also simulated the optical DEMUX characteristics for the time-multiplexed signals by the repetitive pump pulses.

The FD-BPM is useful to obtain the propagation characteristics of single pulse or milti-pulses using the modified nonlinear Schrödinger equation (MNLSE) (Hong et al., 1996 \& Das et al., 2000), simply by changing only the combination of input optical pulses. These are: (1) single pulse propagation (Das et al., 2008), (2) FWM characteristics using two input pulses (Das et al., 2000), (3) optical DENUX using several input pulses (Das et al., 2001), (4) optical phaseconjugation using two input pulses with chirp (Das et al., 2001) and (5) optimum time-delayed FWM characteristics between the two input pump and probe pulses (Das et al., 2007).

\section{Analytical model}

In this section, we briefly discuss the important nonlinear effects in SOAs, mathematical formulation of modified nonlinear Schrödinger equation (MNLSE), finite-difference beam propagation method (FD-BPM) used in the simulation, and nonlinear propagation of solitary pulses in SOAs.

\subsection{Important nonlinear effects in SOAs}

There are several types of "nonlinear effects" in SOAs. Among them, the important four "nonlinear effects" are shown in Fig. 1. These are (i) spectral hole-burning (SHB), (ii) carrier heating $(\mathrm{CH})$, (iii) carrier depletion (CD) and (iv) two-photon absorption (TPA). 

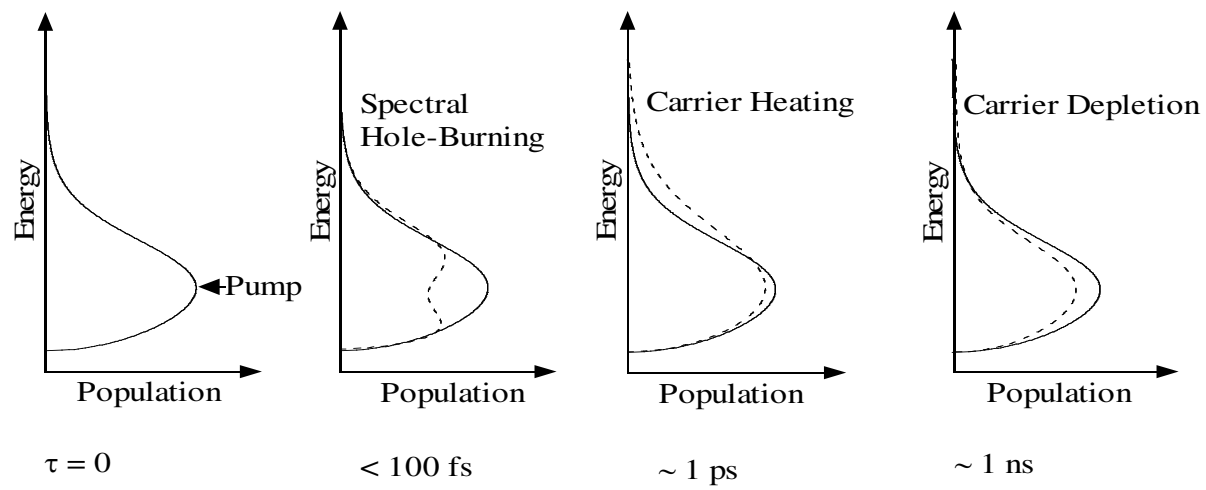

Fig. 1. Important nonlinear effects in SOAs are: (i) spectral hole-burning (SHB) with a life time of $<100 \mathrm{fs}$, (ii) carrier heating $(\mathrm{CH})$ with a life time of $\sim 1 \mathrm{ps}$, (iii) carrier depletion $(\mathrm{CD})$ with a life time is $\sim 1 \mathrm{~ns}$ and (iv) two-photon absorption (TPA).

Figure 1 shows the time-development of the population density in the conduction band after excitation (Das, 2000). The arrow (pump) shown in Fig. 1 is the excitation laser energy. Below the life-time of $100 \mathrm{fs}$, the SHB effect is dominant. SHB occurs when a narrow-band strong pump beam excites the SOA, which has an inhomogeneous broadening. SHB arises due to the finite value of intraband carrier-carrier scattering time ( $50-100 \mathrm{fs})$, which sets the time scale on which a quasi-equilibrium Fermi distribution is established among the carriers in a band. After $\sim 1$ ps, the SHB effect is relaxed and the $\mathrm{CH}$ effect becomes dominant. The process tends to increase the temperature of the carriers beyond the lattice's temperature. The main causes of heating the carriers are (1) the stimulated emission, since it involves the removal of "cold" carriers close to the band edge and (2) the free-carrier absorption, which transfers carriers to high energies within the bands. The "hot"-carriers relax to the lattice temperature through the emission of optical phonons with a relaxation time of $\sim 0.5-1$ ps. The effect of CD remains for about $1 \mathrm{~ns}$. The stimulated electron-hole recombination depletes the carriers, thus reducing the optical gain. The band-to-band relaxation also causes $C D$, with a relaxation time of $\sim 0.2-1$ ns. For ultrashort optical pumping, the two-photon absorption (TPA) effect also becomes important. An atom makes a transition from its ground state to the excited state by the simultaneous absorption of two laser photons. All these nonlinear effects (mechanisms) are taken into account in the simulation and the mathematical formulation of modified nonlinear Schrödinger equation (MNLSE).

\subsection{Mathematical formulation of modified nonlinear schrödinger equation (MNLSE)}

In this subsection, we will briefly explain the theoretical analysis of short optical pulses propagation in SOAs. We start from Maxwell's equations (Agrawal, 1989; Yariv, 1991; Sauter, 1996) and reach the propagation equation of short optical pulses in SOAs, which are governed by the wave equation (Agrawal \& Olsson, 1989) in the frequency domain:

$$
\nabla^{2} \bar{E}(x, y, z, \omega)+\frac{\varepsilon_{r}}{c^{2}} \omega^{2} \bar{E}(x, y, z, \omega)=0
$$


where, $\bar{E}(x, y, z, \omega)$ is the electromagnetic field of the pulse in the frequency domain, $c$ is the velocity of light in vacuum and $\varepsilon_{r}$ is the nonlinear dielectric constant which is dependent on the electric field in a complex form. By slowly varying the envelope approximation and integrating the transverse dimensions we arrive at the pulse propagation equation in SOAs (Agrawal \& Olsson, 1989; Dienes et al., 1996).

$$
\frac{\partial V(\omega, z)}{\partial z}=-i\left\{\frac{\omega}{c}\left[1+\chi_{m}(\omega)+\Gamma \tilde{\chi}(\omega, N)\right]^{1 / 2}-\beta_{0}\right\} V(\omega, z)
$$

where, $V(\omega, z)$ is the Fourier-transform of $V(t, z)$ representing pulse envelope, $\chi_{m}(\omega)$ is the background (mode and material) susceptibility, $\tilde{\chi}(\omega)$ is the complex susceptibility which represents the contribution of the active medium, $N$ is the effective population density, $\beta_{0}$ is the propagation constant. The quantity $\Gamma$ represents the overlap/ confinement factor of the transverse field distribution of the signal with the active region as defined in (Agrawal \& Olsson, 1989).

Using mathematical manipulations (Sauter, 1996; Dienes et al., 1996), including the real part of the instantaneous nonlinear Kerr effect as a single nonlinear index $\mathrm{n}_{2}$ and by adding the two-photon absorption (TPA) term we obtain the MNLSE for the phenomenological model of semiconductor laser and amplifiers (Hong et al., 1996). The following MNLSE (Hong et al., 1996; Das et al., 2000) is used for the simulation of FWM characteristics with solitary probe pulse and optical DEMUX characteristics with multi-probe or pump in SOAs:

$$
\begin{aligned}
& {\left[\frac{\partial}{\partial z}-\frac{i}{2} \beta_{2} \frac{\partial^{2}}{\partial \tau^{2}}+\frac{\gamma}{2}+\left(\frac{\gamma_{2 p}}{2}+i b_{2}\right)|V(\tau, z)|^{2}\right] V(\tau, z)} \\
& =\left\{\frac{1}{2} g_{N}(\tau)\left[\frac{1}{f(\tau)}+i \alpha_{N}\right]+\frac{1}{2} \Delta g_{T}(\tau)\left(1+i \alpha_{T}\right)-\left.i \frac{1}{2} \frac{\partial g(\tau, \omega)}{\partial \omega}\right|_{\omega_{0}} \frac{\partial}{\partial \tau}-\left.\frac{1}{4} \frac{\partial^{2} g(\tau, \omega)}{\partial \omega^{2}}\right|_{\omega_{0}} \frac{\partial^{2}}{\partial \tau^{2}}\right\} V(\tau, z)
\end{aligned}
$$

We introduce the frame of local time $\tau\left(=t-\mathrm{z} / \mathrm{v}_{g}\right)$, which propagates with a group velocity $\mathrm{v}_{g}$ at the center frequency of an optical pulse. A slowly varying envelope approximation is used in (3), where the temporal variation of the complex envelope function is very slow compared with the cycle of the optical field. In (3), $V(\tau, z)$ is the time domain complex envelope function of an optical pulse, $|V(\tau, z)|^{2}$ corresponding to the optical power, and $\beta_{2}$ is the GVD. $\gamma$ is the linear loss, $\gamma_{2 p}$ is the two-photon absorption coefficient, $b_{2}\left(=\omega_{0} n_{2} / c A\right)$ is the instantaneous self-phase modulation term due to the instantaneous nonlinear Kerr effect $n_{2}, \omega_{0}\left(=2 \pi f_{0}\right)$ is the center angular frequency of the pulse, $c$ is the velocity of light in vacuum, $A(=w d / \Gamma)$ is the effective area $(d$ and $w$ are the thickness and width of the active region, respectively and $\Gamma$ is the confinement factor) of the active region.

The saturation of the gain due to the CD is given by (Hong et al., 1996)

$$
g_{N}(\tau)=g_{0} \exp \left(-\frac{1}{W_{s}} \int_{-\infty}^{\tau} e^{-s / \tau_{s}}|V(s)|^{2} d s\right)
$$


where, $g_{N}(\tau)$ is the saturated gain due to $\mathrm{CD}, g_{0}$ is the linear gain, $W_{s}$ is the saturation energy, $\tau_{s}$ is the carrier lifetime.

The SHB function $f(\tau)$ is given by (Hong et al., 1996)

$$
f(\tau)=1+\frac{1}{\tau_{s h b} P_{\text {shb }}} \int_{-\infty}^{+\infty} u(s) e^{-s / \tau_{\text {shb }}}|V(\tau-s)|^{2} d s
$$

where, $f(\tau)$ is the SHB function, $P_{\text {shb }}$ is the SHB saturation power, $\tau_{\text {shb }}$ is the SHB relaxation time, and $\alpha_{N}$ and $\alpha_{T}$ are the linewidth enhancement factor associated with the gain changes due to the $\mathrm{CD}$ and $\mathrm{CH}$.

The resulting gain change due to the $\mathrm{CH}$ and TPA is given by (Hong et al., 1996)

$$
\begin{aligned}
\Delta g_{T}(\tau) & =-h_{1} \int_{-\infty}^{+\infty} u(s) e^{-s / \tau_{c h}}\left(1-e^{-s / \tau_{s h b}}\right)|V(\tau-s)|^{2} d s \\
& -h_{2} \int_{-\infty}^{+\infty} u(s) e^{-s / \tau_{c h}}\left(1-e^{-s / \tau_{s h b}}\right)|V(\tau-s)|^{4} d s
\end{aligned}
$$

where, $\Delta g_{T}(\tau)$ is the resulting gain change due to the CH and TPA, $u(\mathrm{~s})$ is the unit step function, $\tau_{c h}$ is the $\mathrm{CH}$ relaxation time, $h_{1}$ is the contribution of stimulated emission and freecarrier absorption to the $\mathrm{CH}$ gain reduction and $h_{2}$ is the contribution of two-photon absorption.

The dynamically varying slope and curvature of the gain plays a shaping role for pulses in the sub-picosecond range. The first and second order differential net (saturated) gain terms are (Hong et al., 1996),

$$
\begin{gathered}
\left.\frac{\partial g(\tau, \omega)}{\partial \omega}\right|_{\omega_{0}}=A_{1}+B_{1}\left[g_{0}-g\left(\tau, \omega_{0}\right)\right] \\
\left.\frac{\partial^{2} g(\tau, \omega)}{\partial \omega^{2}}\right|_{\omega_{0}}=A_{2}+B_{2}\left[g_{0}-g\left(\tau, \omega_{0}\right)\right] \\
g\left(\tau, \omega_{0}\right)=g_{N}\left(\tau, \omega_{0}\right) / f(\tau)+\Delta g_{T}\left(\tau, \omega_{0}\right)
\end{gathered}
$$

where, $A_{1}$ and $A_{2}$ are the slope and curvature of the linear gain at $\omega_{0}$, respectively, while $B_{1}$ and $B_{2}$ are constants describing changes in $A_{1}$ and $A_{2}$ with saturation, as given in (7) and (8).

The gain spectrum of an SOA is approximated by the following second-order Taylor expansion in $\Delta \omega$ :

$$
g(\tau, \omega)=g\left(\tau, \omega_{0}\right)+\left.\Delta \omega \frac{\partial g(\tau, \omega)}{\partial \omega}\right|_{\omega_{0}}+\left.\frac{(\Delta \omega)^{2}}{2} \frac{\partial^{2} g(\tau, \omega)}{\partial \omega^{2}}\right|_{\omega_{0}}
$$


The coefficients $\left.\frac{\partial g(\tau, \omega)}{\partial \omega}\right|_{\omega_{0}}$ and $\left.\frac{\partial^{2} g(\tau, \omega)}{\partial \omega^{2}}\right|_{\omega_{0}}$ are related to $A_{1}, B_{1}, A_{2}$ and $B_{2}$ by (7) and (8).

Here we assumed the same values of $A_{1}, B_{1}, A_{2}$ and $B_{2}$ as in (Hong et al., 1996) for an AlGaAs/GaAs bulk SOA.

The time derivative terms in (3) have been replaced by the central-difference approximation in order to simulate this equation by the FD-BPM (Das et al., 2000). In simulation, the parameter of bulk SOAs (AlGaAs/GaAs, double heterostructure) with a wavelength of 0.86 $\mu \mathrm{m}$ (Hong et al., 1996) is used and the SOA length is $350 \mu \mathrm{m}$. The input pulse shape is sech ${ }^{2}$ and is Fourier transform-limited.
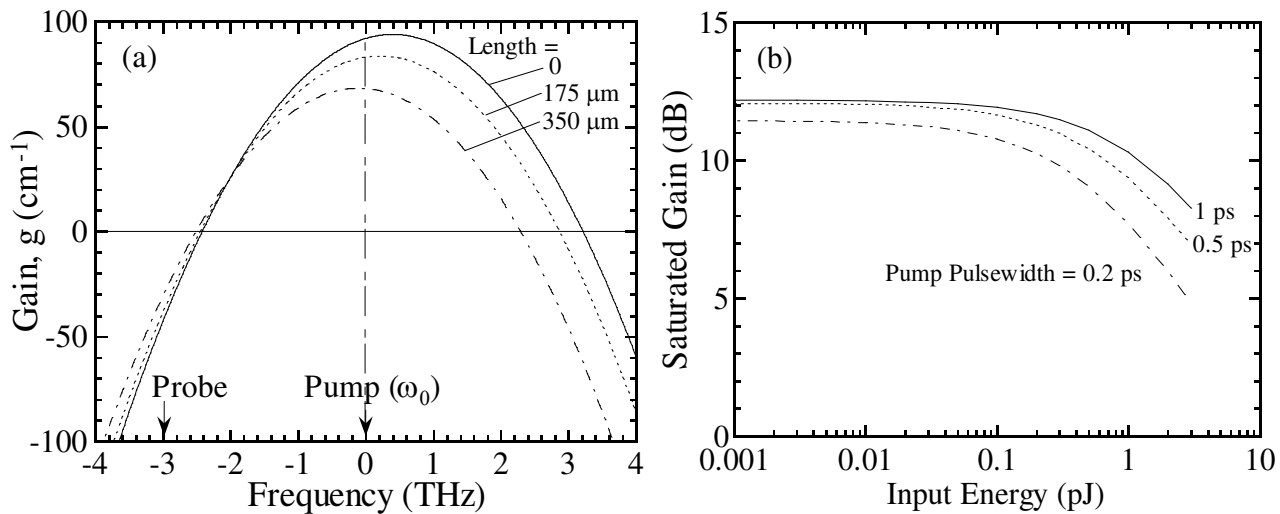

Fig. 2. (a) The gain spectra given by the second-order Taylor expansion about the center frequency of the pump pulse $\omega_{0}$. The solid line shows the unsaturated gain spectrum (length: 0 $\mu \mathrm{m})$, the dotted and the dashed-dotted lines are a saturated gain spectrum at $175 \mu \mathrm{m}$ and 350 $\mu \mathrm{m}$, respectively. Here, the input pump pulse pulsewidth is 1 ps and pulse energy is $1 \mathrm{pJ}$. (b) Saturated gain versus the input pump pulse energy characteristics of the SOA. The saturation energy decreases with decreasing the input pump pulsewidth. The SOA length is $350 \mu \mathrm{m}$. The input pulsewidths are $0.2 \mathrm{ps}, 0.5 \mathrm{ps}$, and 1 ps respectively, and a pulse energy of $1 \mathrm{pJ}$.

The gain spectra of SOAs are very important for obtaining the propagation and wave mixing (FWM and optical DEMUX between the input pump and probe pulses) characteristics of short optical pulses. Figure 2(a) shows the gain spectra given by a second-order Taylor expansion about the pump pulse center frequency $\omega_{0}$ with derivatives of $g(\tau, \omega)$ by $(7)$ and (8) (Das et al., 2000). In Fig. 2(a), the solid line represents an unsaturated gain spectrum (length: $0 \mu \mathrm{m}$ ), the dotted line represents a saturated gain spectrum at the center position of the SOA (length: 175 $\mu \mathrm{m})$, and the dashed-dotted line represents a saturated gain spectrum at the output end of the SOA (length: $350 \mu \mathrm{m}$ ), when the pump pulsewidth is 1 ps and input energy is $1 \mathrm{pJ}$. These gain spectra were calculated using (1), because, the waveforms of optical pulses depend on the propagation distance (i.e., the SOA length). The spectra of these pulses were obtained by Fourier transformation. The "local" gains at the center frequency at $z=0,175$, and $350 \mu \mathrm{m}$ were obtained from the changes in the pulse intensities at the center frequency at around those positions (Das et al., 2001). The gain at the center frequency in the gain spectrum was 
approximated by the second-order Taylor expression series. As the pulse propagates in the SOA, the pulse intensity increases due to the gain of the SOA. The increase in pulse intensity reduces the gain, and the center frequency of the gain shifts to lower frequencies. The pump frequency is set to near the gain peak, and linear gain $g_{0}$ is $92 \mathrm{~cm}$ at $\omega_{0}$. The probe frequency is set $-3 \mathrm{THz}$ from $\omega_{0}$ for the calculations of FWM characteristics as described below, and the linear gain $g_{0}$ is $-42 \mathrm{~cm}$ at this frequency. Although the probe frequency lies outside the gain bandwidth, we selected a detuning of $3 \mathrm{THz}$ in this simulation because the FWM signal must be spectrally separated from the output of the SOA. As will be shown later, even for this large degree of detuning, the FWM signal pulse and the pump pulse spectrally overlap when the pulsewidths become short ( $<0.5 \mathrm{ps}$ ) (Das et al., 2001). The gain bandwidth is about the same as the measured value for an AlGaAs/GaAs bulk SOA (Seki et al., 1981). If an InGaAsP/InP bulk SOA is used we can expect much wider gain bandwidth (Leuthold et al., 2000). With a decrease in the carrier density, the gain decreases and the peak position is shifted to a lower frequency because of the band-filling effect. Figure 2(b) shows the saturated gain versus input pump pulse energy characteristics of the SOA. When the input pump pulsewidth decreases then the small signal gain decreases due to the spectral limit of the gain bandwidth. For the case, when the input pump pulsewidth is short (very narrow, such as $200 \mathrm{fs}$ or lower), the gain saturates at small input pulse energy (Das et al., 2000). This is due to the $\mathrm{CH}$ and SHB with the fast response.

Initially, the MNLSE was used by (Hong et al., 1996) for the analysis of "solitary pulse" propagation in an SOA. We used the same MNLSE for the simulation of FWM and optical DEMUX characteristics in SOA using the FD-BPM. Here, we have introduced a complex envelope function $\mathrm{V}(\tau, 0)$ at the input side of the SOA for taking into account the two (pump and probe) or more (multi-pump or probe) pulses.

\subsection{Finite-difference beam propagation method (FD-BPM)}

To solve a boundary value problem using the finite-differences method, every derivative term appearing in the equation, as well as in the boundary conditions, is replaced by the central differences approximation. Central differences are usually preferred because they lead to an excellent accuracy (Conte \& Boor, 1980). In the modeling, we used the finitedifferences (central differences) to solve the MNLSE for this analysis.

Usually, the fast Fourier transformation beam propagation method (FFT-BPM) (Okamoto, 1992; Brigham, 1988) is used for the analysis of the optical pulse propagation in optical fibers by the successive iterations of the Fourier transformation and the inverse Fourier transformation. In the FFT-BPM, the linear propagation term (GVD term) and phase compensation terms (other than GVD, 1st and 2nd order gain spectrum terms) are separated in the nonlinear Schrödinger equation for the individual consideration of the time and frequency domain for the optical pulse propagation. However, in our model, equation (3) includes the dynamic gain change terms, i.e., the 1st and 2 nd order gain spectrum terms which are the last two terms of the right-side in equation (3). Therefore, it is not possible to separate equation (3) into the linear propagation term and phase compensation term and it is quite difficult to calculate equation (3) using the FFT-BPM. For this reason, we used the FD-BPM (Chung \& Dagli, 1990; Conte \& Boor, 1980; Das et al., 2000; Razaghi et al., 2009). If we replace the time derivative terms of equation (3) by the below central-difference approximation, equation (11), and integrate equation (3) with the small propagation step $\Delta z$, we obtain the tridiagonal simultaneous matrix equation (12) 


$$
\frac{\partial}{\partial \tau} V_{k}=\frac{V_{k+1}-V_{k-1}}{2 \Delta \tau}, \frac{\partial^{2}}{\partial \tau^{2}} V_{k}=\frac{V_{k+1}-2 V_{k}+V_{k-1}}{\Delta \tau^{2}}
$$

where, $V_{k}=V\left(\tau_{k}\right), V_{k+1}=V\left(\tau_{k}+\Delta \tau\right)$, and $V_{k-1}=V\left(\tau_{k}-\Delta \tau\right)$

$$
\begin{array}{r}
-a_{k}(z+\Delta z) V_{k-1}(z+\Delta z)+\left\{1-b_{k}(z+\Delta z)\right\} V_{k}(z+\Delta z)-c_{k}(z+\Delta z) V_{k+1}(z+\Delta z) \\
=a_{k}(z) V_{k-1}(z)+\left\{1+b_{k}(z)\right\} V_{k}(z)+c_{k}(z) V_{k+1}(z)
\end{array}
$$

where, $k=1,2,3, \ldots \ldots \ldots . . ., n$ and

$$
\begin{aligned}
a_{k}(z)= & \frac{\Delta z}{2}\left[\frac{i \beta_{2}}{2 \Delta \tau^{2}}+\left.i \frac{1}{4 \Delta \tau} \frac{\partial g(\tau, \omega, z)}{\partial \omega}\right|_{\omega_{0}, \tau_{k}}-\left.\frac{1}{4 \Delta \tau^{2}} \frac{\partial^{2} g(\tau, \omega, z)}{\partial \omega^{2}}\right|_{\omega_{0}, \tau_{k}}\right] \\
b_{k}(z)= & -\frac{\Delta z}{2}\left[\frac{i \beta_{2}}{\Delta \tau^{2}}+\frac{\gamma}{2}+\left(\frac{\gamma_{2 p}}{2}+i b_{2}\right)\left|V_{k}(z)\right|^{2}-\frac{1}{2} g_{N}\left(\tau_{k}, \omega_{0}, z\right)\left(1+i \alpha_{N}\right)\right. \\
& \left.-\frac{1}{2} \Delta g_{T}\left(\tau_{k}, \omega_{0}, z\right)\left(1+i \alpha_{T}\right)-\left.\frac{1}{2 \Delta \tau^{2}} \frac{\partial^{2} g(\tau, \omega, z)}{\partial \omega^{2}}\right|_{\omega_{0}, \tau_{k}}\right] \\
c_{k}(z)= & \frac{\Delta z}{2}\left[\frac{i \beta_{2}}{2 \Delta \tau^{2}}-\left.i \frac{1}{4 \Delta \tau} \frac{\partial g(\tau, \omega, z)}{\partial \omega}\right|_{\omega_{0}, \tau_{k}}-\left.\frac{1}{4 \Delta \tau^{2}} \frac{\partial^{2} g(\tau, \omega, z)}{\partial \omega^{2}}\right|_{\omega_{0}, \tau_{k}}\right]
\end{aligned}
$$

where, $\Delta \tau$ is the sampling time and $n$ is the number of sampling. If we know $V_{k}(z)$, $(k=1,2,3, \ldots \ldots \ldots, n)$ at the position $z$, we can calculate $V_{k}(z+\Delta z)$ at the position of $z+\Delta z$ which is the propagation of a step $\Delta z$ from position $z$, by using equation (12). It is not possible to directly calculate equation (12) because it is necessary to calculate the left-side terms $a_{k}(z+\Delta z), b_{k}(z+\Delta z)$, and $c_{k}(z+\Delta z)$ of equation (12) from the unknown $V_{k}(z+\Delta z)$. Therefore, we initially defined $a_{k}(z+\Delta z) \equiv a_{k}(z), b_{k}(z+\Delta z) \equiv b_{k}(z)$, and $c_{k}(z+\Delta z) \equiv c_{k}(z)$ and obtained $V_{k}^{(0)}(z+\Delta z)$, as the zeroth order approximation of $V_{k}(z+\Delta z)$ by using equation (12). We then substituted $V_{k}^{(0)}(z+\Delta z)$ in equation (12) and obtained $V_{k}^{(1)}(z+\Delta z)$ as the first order approximation of $V_{k}(z+\Delta z)$ and finally obtained the accurate simulation results by the iteration as used in (Brigham, 1988; Chung \& Dagli, 1990; Das et al., 2000; Razaghi et al., 2009).

Figure 3 shows a simple schematic diagram of the FD-BPM in time domain. Here, $\tau\left(=t-z / v_{g}\right)$ is the local time, which propagates with the group velocity $v_{g}$ at the center frequency of an optical pulse and $\Delta \tau$ is the sampling time. $\mathrm{z}$ is the propagation direction and $\Delta \mathrm{z}$ is the propagation step. With this procedure, we used up to 3-rd time iteration for more accuracy of the simulations. 


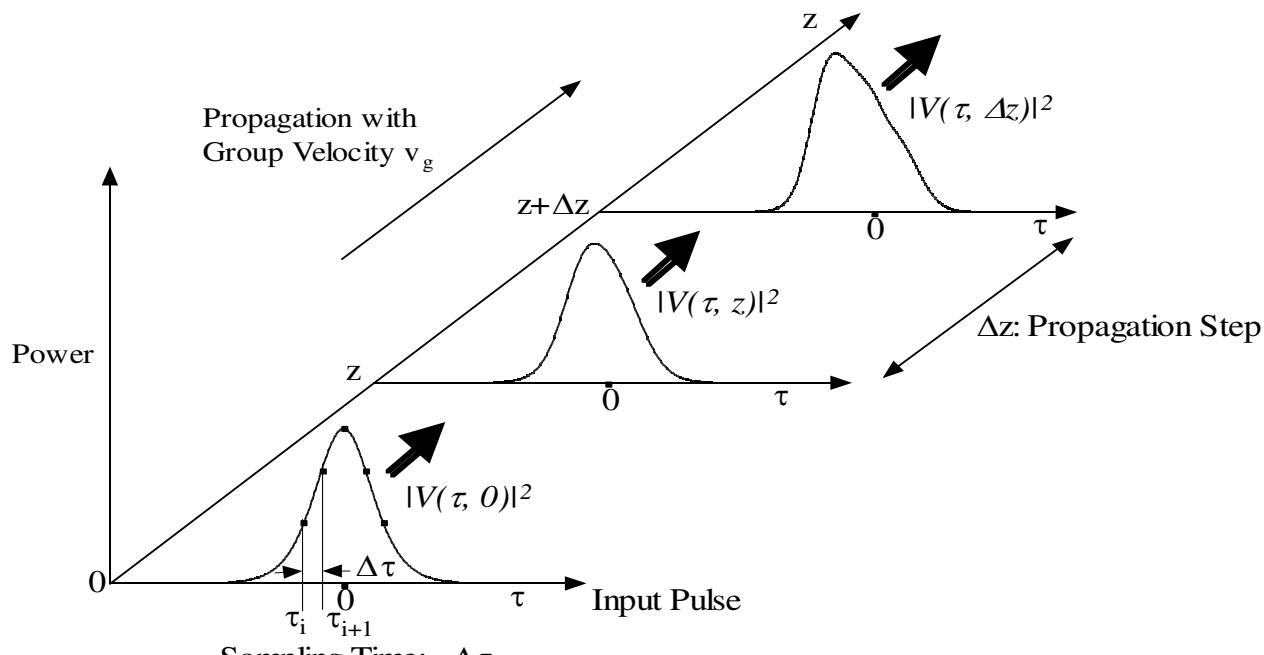

Sampling Time: $\Delta \tau$

Fig. 3. A simple schematic diagram of FD-BPM in the time domain, where, $\tau\left(=t-z / v_{g}\right)$ is the local time, which propagates with the group velocity $v_{g}$ at the center frequency of an optical pulse and $\Delta \tau$ is the sampling time, and $z$ is the propagation direction and $\Delta z$ is the propagation step.

The FD-BPM (Conte \& Boor, 1980; Chung \& Dagli, 1990; Das et al., 2000; Razaghi et al., 2009a $\& 2009 b$ ) is used for the simulation of several important characteristics, namely, (1) single pulse propagation in SOAs (Das et al., 2008; Razaghi et al., 2009a \& 2009b), (2) two input pulses propagating in SOAs (Das et al., 2000; Connelly et al., 2008), (3) Optical DEMUX characteristics of multi-probe or pump input pulses based on FWM in SOAs (Das et al., 2001), (4) Optical phase-conjugation characteristics of picosecond FWM signal in SOAs (Das et al., 2001), and (5) FWM conversion efficiency with optimum time-delays between the input pump and probe pulses (Das et al., 2007).

\subsection{Nonlinear optical pulse propagation model in SOAs}

Nonlinear optical pulse propagation in SOAs has drawn considerable attention due to its potential applications in optical communication systems, such as a wavelength converter based on FWM and switching. The advantages of using SOAs include the amplification of small (weak) optical pulses and the realization of high efficient FWM characteristics.

For the analysis of optical pulse propagation in SOAs using the FD-BPM in conjunction with the MNLSE, where several parameters are taken into account, namely, the group velocity dispersion, self-phase modulation (SPM), and TPA, as well as the dependencies on the CD, $\mathrm{CH}, \mathrm{SHB}$ and their dispersions, including the recovery times in an SOA (Hong et al., 1996). We also considered the gain spectrum (as shown in Fig. 2). The gain in an SOA was dynamically changed depending on values used for the carrier density and carrier temperature in the propagation equation (i.e., MNLSE). 
Initially, (Hong et al., 1996) used the MNLSE for the simulation of optical pulse propagation in an SOA by FFT-BPM (Okamoto, 1992; Brigham, 1988) but the dynamic gain terms were changing with time. The FD-BPM is capable to simulate the optical pulse propagation taking into consideration the dynamic gain terms in SOAs (Das et al., 2000 \& 2007; Razaghi et al., 2009a \& 2009b; Aghajanpour et al., 2009). We used the MNLSE for nonlinear optical pulse propagation in SOAs by the FD-BPM (Chung \& Dagli, 1990; Conte \& Boor, 1980). We used the FD-BPM for the simulation of optical DEMUX characteristics in SOAs with the multiinput pump and probe pulses.

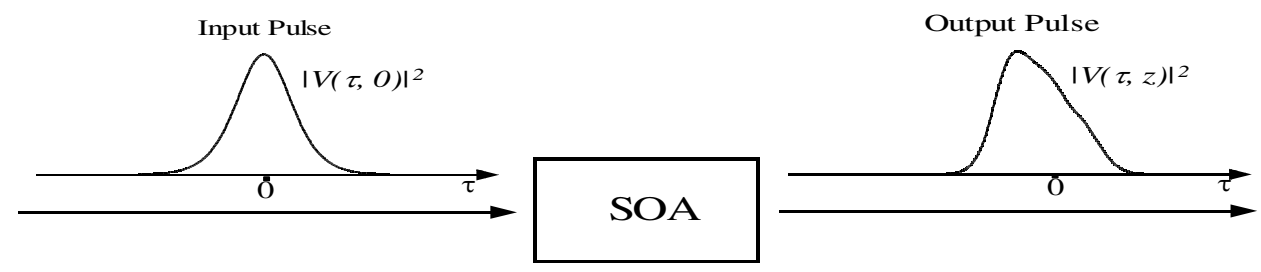

Fig. 4. A simple schematic diagram for the simulation of nonlinear single pulse propagation in SOA. Here, $|V(\tau, 0)|^{2}$ is the input $(\mathrm{z}=0)$ pulse intensity and $|V(\tau, z)|^{2}$ is the output pulse intensity (after propagating a distance $\mathrm{z}$ ) of SOA.

Figure 4 illustrates a simple model for the simulation of nonlinear optical pulse propagation in an SOA. An optical pulse is injected into the input side of the SOA $(z=0)$. Here, $\tau$ is the local time, $|V(\tau, 0)|^{2}$ is the intensity (power) of input pulse $(z=0)$ and $|V(\tau, z)|^{2}$ is the intensity (power) of the output pulse after propagating a distance $\mathbf{z}$ at the output side of SOA. We used this model to simulate FWM (with single probe) and DEMUX (with multi-bit pump or probe pulses) characteristics in SOAs.

\section{FWM characteristics in SOAs with the solitary probe pulse}

In this section, we will discuss the FWM characteristics with the solitary probe pulse in SOAs. When two optical pulses with different central frequencies $f_{p}$ (pump) and $f_{q}$ (probe) are injected simultaneously into the SOA, an FWM signal is generated at the output of the SOA at a frequency of $2 f_{p}-f_{q}$ (as shown in Fig. 5). For the analysis (simulation) of FWM characteristics, the total input pump and probe pulse, $V_{\text {in }}(\tau)$, is given by the following equation

$$
V_{i n}(\tau)=V_{p}(\tau)+V_{q}(\tau) \exp (-i 2 \pi \Delta f \tau)
$$

where, $V_{p}(\tau)$ and $V_{q}(\tau)$ are the complex envelope functions of the input pump and probe pulses respectively, $\tau\left(=t-z / v_{g}\right)$ is the local time that propagates with group velocity $v_{g}$ at the center frequency of an optical pulse, $\Delta f$ is the detuning frequency between the input pump and probe pulses and expressed as $\Delta f=f_{p}-f_{q}$. Using the complex envelope function of (16), we solved the MNLSE and obtained the combined spectrum of the amplified pump, probe and the generated FWM signal at the output of SOA. 


\begin{tabular}{|c|c|c|c|}
\hline Name of the Parameters & Symbols & Values & Units \\
\hline Length of SOA & $\mathrm{L}$ & 350 & $\mu \mathrm{m}$ \\
\hline Effective area & $\mathrm{A}$ & 5 & $\mu \mathrm{m}^{2}$ \\
\hline Center frequency of the pulse & $\mathrm{f}_{0}$ & 349 & $\mathrm{THz}$ \\
\hline Linear gain & $\mathrm{g}_{0}$ & 92 & $\mathrm{~cm}^{-1}$ \\
\hline Group velocity dispersion & $\beta_{2}$ & 0.05 & $\mathrm{ps}^{2} \mathrm{~cm}^{-1}$ \\
\hline Saturation energy & $\mathrm{W}_{\mathrm{s}}$ & 80 & $\mathrm{pJ}$ \\
\hline Linewidth enhancement factor due to the CD & $\alpha_{N}$ & 3.1 & \\
\hline Linewidth enhancement factor due to the $\mathrm{CH}$ & $\alpha_{\mathrm{T}}$ & 2.0 & \\
\hline $\begin{array}{l}\text { The contribution of stimulated emission and FCA to } \\
\text { the } \mathrm{CH} \text { gain reduction }\end{array}$ & $\mathrm{h}_{1}$ & 0.13 & $\mathrm{~cm}^{-1} \mathrm{pJ}^{-1}$ \\
\hline The contribution of TPA & $\mathrm{h}_{2}$ & 126 & fs $\mathrm{cm}^{-1} \mathrm{pJ}^{-2}$ \\
\hline Carrier lifetime & $\tau_{\mathrm{s}}$ & 200 & ps \\
\hline $\mathrm{CH}$ relaxation time & $\tau_{\mathrm{ch}}$ & 700 & fs \\
\hline SHB relaxation time & $\tau_{\mathrm{shb}}$ & 60 & fs \\
\hline SHB saturation power & $P_{\text {shb }}$ & 28.3 & $\mathrm{~W}$ \\
\hline Linear loss & $\gamma$ & 11.5 & $\mathrm{~cm}^{-1}$ \\
\hline Instantaneous nonlinear Kerr effect & $\mathrm{n}_{2}$ & -0.70 & $\mathrm{~cm}^{2} \mathrm{TW}^{-1}$ \\
\hline TPA coefficient & $\gamma_{2 p}$ & 1.1 & $\mathrm{~cm}^{-1} \mathrm{~W}^{-1}$ \\
\hline $\begin{array}{l}\text { Parameters describing second-order Taylor expansion } \\
\text { of the dynamically gain spectrum }\end{array}$ & $\begin{array}{l}\mathrm{A}_{1} \\
\mathrm{~B}_{1} \\
\mathrm{~A}_{2} \\
\mathrm{~B}_{2}\end{array}$ & $\begin{array}{c}0.15 \\
-80 \\
-60 \\
0\end{array}$ & $\begin{array}{c}\text { fs } \mu \mathrm{m}^{-1} \\
\mathrm{fs} \\
\mathrm{fs}^{2} \mu \mathrm{m}^{-1} \\
\mathrm{fs}^{2}\end{array}$ \\
\hline
\end{tabular}

Table 1. Simulation parameters of a bulk SOA (AlGaAs/GaAs, double heterostructure) (Hong et al., 1996; Das et al., 2000).

For the simulations, we used the parameters of a bulk SOA (AlGaAs/GaAs, double heterostructure) at a wavelength of $0.86 \mu \mathrm{m}$. The parameters are listed in Table 1 (Hong et al., 1996). The length of the SOA was assumed to be $350 \mu \mathrm{m}$. All the results were obtained for a propagation step $\Delta \mathrm{z}$ of $5 \mu \mathrm{m}$. We confirmed that for any step size less than $5 \mu \mathrm{m}$ the simulation results were almost identical (i.e., independent of the step size).

For the simulation of optical DEMUX characteristics in SOAs, we have started with the simulation of FWM characteristics for solitary probe pulse's. Figure 5 shows a simple schematic diagram illustrating the simulation of the FWM characteristics in an SOA between short optical pulses. In SOAs, the FWM signal is generated by mixing between the input pump and probe pulses, whose frequency appears at the symmetry position of the probe pulse with respect to the pump. We have selected the detuning frequency between the input pump and probe pulses to $+3 \mathrm{THz}$. The generated FWM signal is filtered using an optical narrow bandpass filter from the optical output spectrum containing the pump and probe signal. Here, the pass-band of the filter is set to be from $+2 \mathrm{THz}$ to $+4 \mathrm{THz}$, i.e., a bandwidth of $2 \mathrm{THz}$ is used. The shape of the pass-band was assumed to be rectangular. The solid line represents for a short pump pulsewidths and the dotted line represents for a wider pump pulsewidths. For a wider pulsewidth, the pump peak intensity decreases, spectral peak intensity increases, and the FWM signal peak intensity decreases as shown in the figure when the input pulse energy is kept constant. 


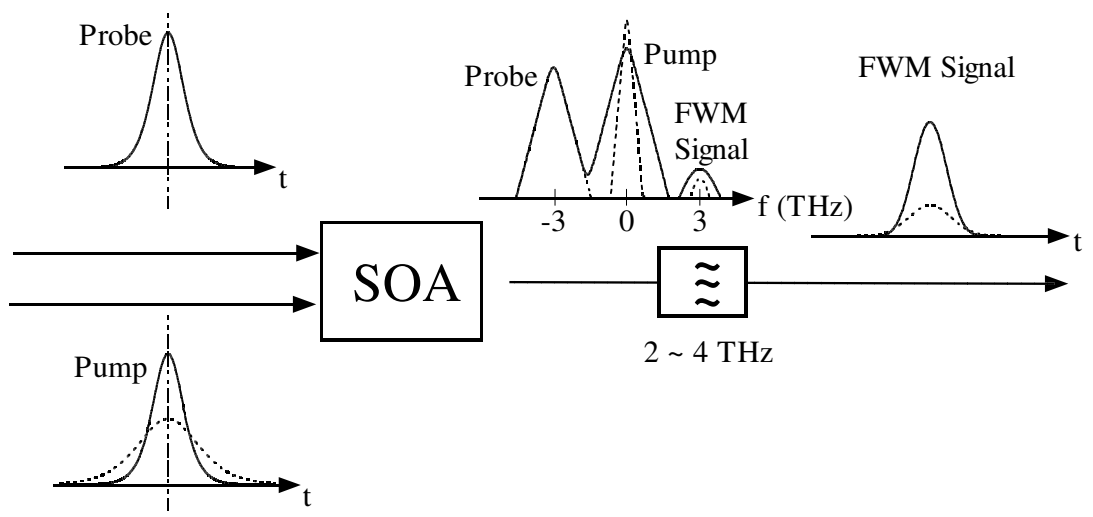

Fig. 5. A simple schematic diagram for the simulation of FWM characteristics for solitary probe pulse for the optimization of the input pump pulsewidth. Here, the input pump pulsewidth is varied.

Figure 6(a) shows the calculated output spectra of the SOA. The solid and dashed curves show the output spectra with pump and probe pulses and with only a pump pulse, respectively. The pump pulsewidths are $1 \mathrm{ps}, 0.5 \mathrm{ps}$, and $0.2 \mathrm{ps}$ and the probe pulsewidth is 1 ps. The input pump and probe pulse energies are $1 \mathrm{pJ}$ and $10 \mathrm{fJ}$, respectively. For a pump pulsewidth of 1 ps, the spectral peaks of the pump, probe, and FWM signals are clearly separated. The FWM signal can be obtained by the spectral filtering whose bandwidth is from $+2 \mathrm{THz}$ to $+4 \mathrm{THz}$, which is shown in the figure by the arrow. With the decrease in the pump pulsewidth, the pump spectral width is broadened and it becomes difficult to extract the FWM signal using the optical filter due to the spectral overlap. For the shorter pump pulsewidth less than $0.5 \mathrm{ps}$, the FWM signals are not clearly observed.

Figure 6(b) shows the temporal waveforms of the output signals after filtering. The solid and dashed curves show the waveforms with probe and without probe pulses, respectively. The contrast between the output power with probe and without probe pulses for a pump pulsewidth of $1 \mathrm{ps}$ is larger than that for the shorter pump pulsewidths. This is due to the strong overlap between pump pulse of 0.5 ps and 0.2 ps and the FWM signal in the frequency domain. For an input pump pulsewidth of 1 ps, a FWM signal pulsewidth of 0.73 ps is narrower than the input pump pulsewidth. This is due to the fact that the FWM signal intensity is proportional to $I_{p}^{2} I_{q}$ i.e., $I_{F W M} \propto I_{p}^{2} I_{q}$ as reported (Das et al., 2000). Here, $I_{F W M}$ is the FWM signal intensity, $I_{p}$ is the pump pulse intensity, and $I_{q}$ is the probe pulse intensity. For input pump pulsewidths of 0.5 ps and $0.2 \mathrm{ps}$, the optical bandpass filter broadens the FWM signal pulsewidth due to the limitation in the frequency domain. Then, FWM signal pulsewidths of $0.57 \mathrm{ps}$ and $0.55 \mathrm{ps}$ become broader than the input pump pulsewidths. By this filtering, the energies of the transform-limited sech ${ }^{2}$ pulses with pulsewidths of 1 ps, 0.73 ps, 0.5 ps, and 0.2 ps are reduced by $0.002 \%, 0.05 \%, 0.7 \%$, and $19 \%$, respectively. The peak powers are decreased by $0.86 \%, 4 \%, 14 \%$, and $63 \%$, respectively. The pulsewidth of 0.73 ps corresponds the that of the FWM pulse among 1 ps pump and 1 ps probe pulses (Fig. 6(b)). Therefore, the waveform distortion by this filtering is negligibly small for the FWM pulses among 1 ps pump and 1 ps probe pulses. 

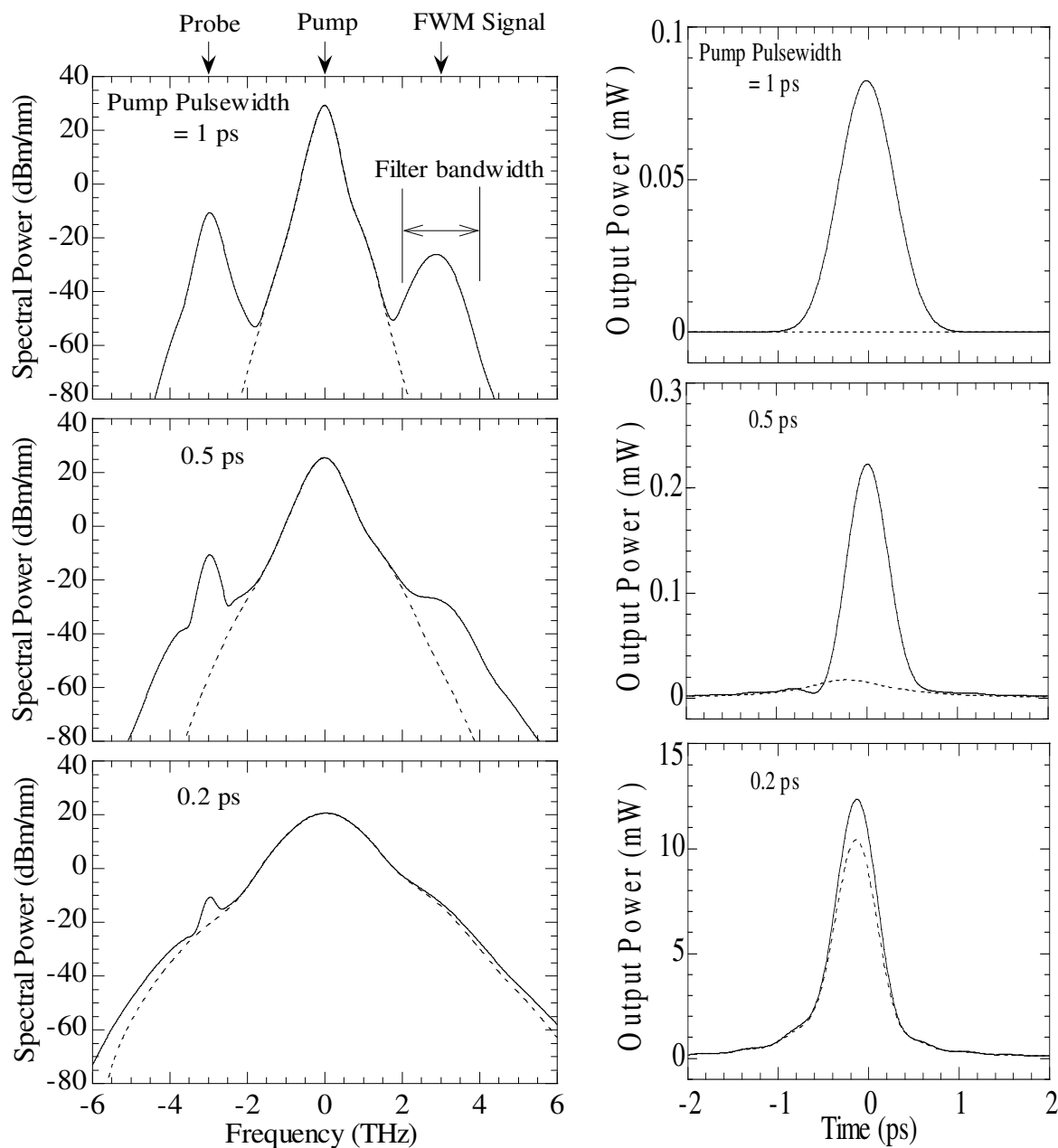

(a)

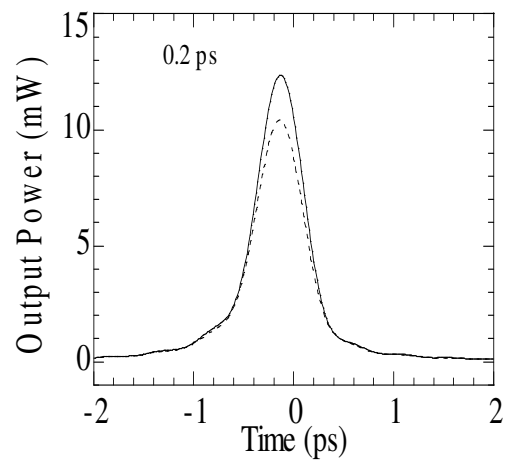

(b)

Fig. 6. (a) Output spectra of the SOA before filtering. The solid and dashed curves show the output spectra with pump and probe pulses and with only a pump pulse, respectively. (b) Output pulse waveforms after filtering from $+2 \sim+4 \mathrm{THz}$. The solid and dashed curves show the output pulse waveforms with pump and probe pulses and with only a pump pulse, respectively. Here, the input pump and probe pulse energies are $1 \mathrm{pJ}$ and $10 \mathrm{fJ}$, respectively. The input probe pulsewidth is $1 \mathrm{ps}$. 


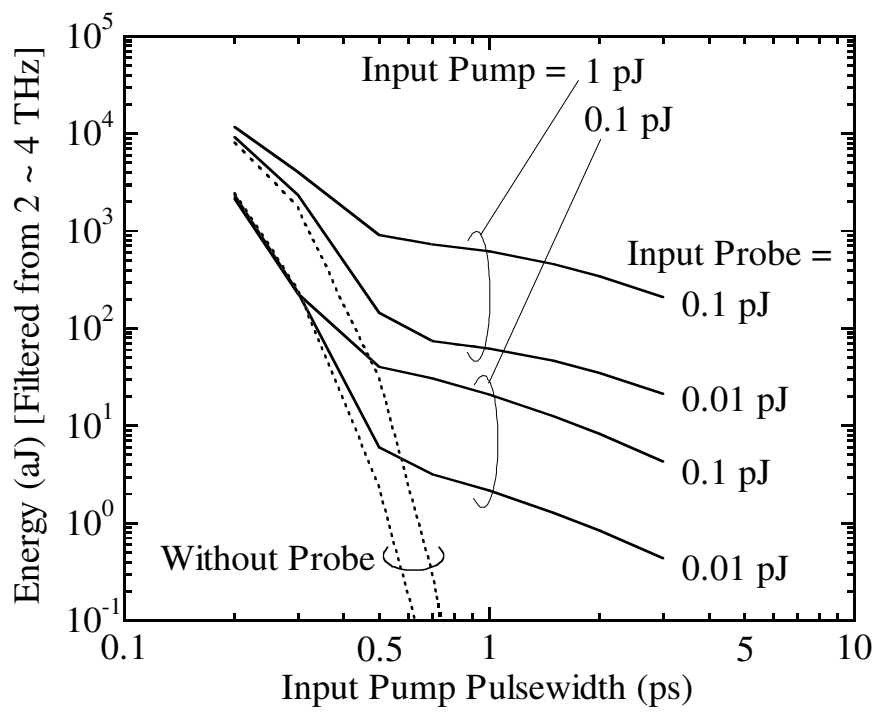

Fig. 7. The energy, which is filtered from the +2 to $+4 \mathrm{THz}$ component of the output spectrum, against the input pump pulsewidth. The input probe pulse energies are $0.01 \mathrm{pJ}$, and $0.1 \mathrm{pJ}$, and pulsewidth is $1 \mathrm{ps}$. The input pump energies are $0.1 \mathrm{pJ}$ and $1 \mathrm{pJ}$. The solid lines represent the FWM signal energy with the input probe pulse and the dashed lines represent the FWM signal energy without input probe pulse.

Figure 7 shows the energy, which is filtered from the +2 to $+4 \mathrm{THz}$ component of the output spectrum (as shown in Fig. 6(a)) versus the input pump pulsewidth characteristics. The solid and dashed curves show the output pulse energy with and without probe pulses, respectively. Here, the input probe pulse energies were set to be $0.01 \mathrm{pJ}$, and $0.1 \mathrm{pJ}$ with a pulsewidth of $1 \mathrm{ps}$ and the input pump pulse energies were set to be $0.1 \mathrm{pJ}$ and $1 \mathrm{pJ}$. With the decrease of pump pulsewidth, the output energy increases, while the differences between the output energy with probe pulses and without probe pulses decrease because of the overlap of the pump and the FWM signal in the spectral domain. Therefore, the regular DEMUX operation is not obtained for the pump pulsewidth of less than $0.5 \mathrm{ps}$.

Figure 8(a) shows the ON-OFF ratio and the FWM conversion efficiency characteristics. Here, the "ON-OFF ratio" is defined as the ratio of the output energy having a spectral component of $+2 \sim+4 \mathrm{THz}$ with the probe pulse to without the probe pulse. Therefore, in the ideal case, the output energy with the probe pulse corresponds to the FWM signal energy and the output energy without the probe pulse becomes zero. The larger ON-OFF ratio is preferable in the DEMUX operations. We have assumed that the ON-OFF ratio > 20 $\mathrm{dB}$ is acceptable for a practical DEMUX operation. To obtain the enough ON-OFF ratio, the pump pulsewidth should be wider than $0.8 \mathrm{ps}$ for input pump pulse energies of $0.1 \mathrm{pJ}$ and 1 pJ. Fig. 8(b) shows the FWM conversion efficiency increases with the increase of input pump pulse energy. The FWM signal intensity is proportional to the square of the input pump intensity (Das et al., 2000). Therefore, the FWM conversion efficiency increases about $20 \mathrm{~dB}$ for the increase in a pump pulse energy of $10 \mathrm{~dB}$ in the region where the enough ON-OFF ratio is obtained. For the narrower pump pulsewidth, the nominal FWM conversion 
efficiency increases. However, the pump pulses narrower than $0.8 \mathrm{ps}$ are not suitable for a practical DEMUX operation, which is due to the low ON-OFF ratio. To improve the ONOFF ratio, one possible method is to increase the detuning for decreasing the spectral overlap between the pump and the FWM signal. However, in our simulation using the parameters for typical SOAs working in a $0.86 \mu \mathrm{m}$ region, the increasing in the detuning is not practical because of the limited gain bandwidth of the SOAs. It is necessary to use the SOA with wider gain bandwidth for FWM among shorter pulses. One good candidate for obtaining wider gain bandwidth is to use the SOAs with staggered thickness multiple quantum wells (Mikami et al.,1991; Gingrich et al., 1997).

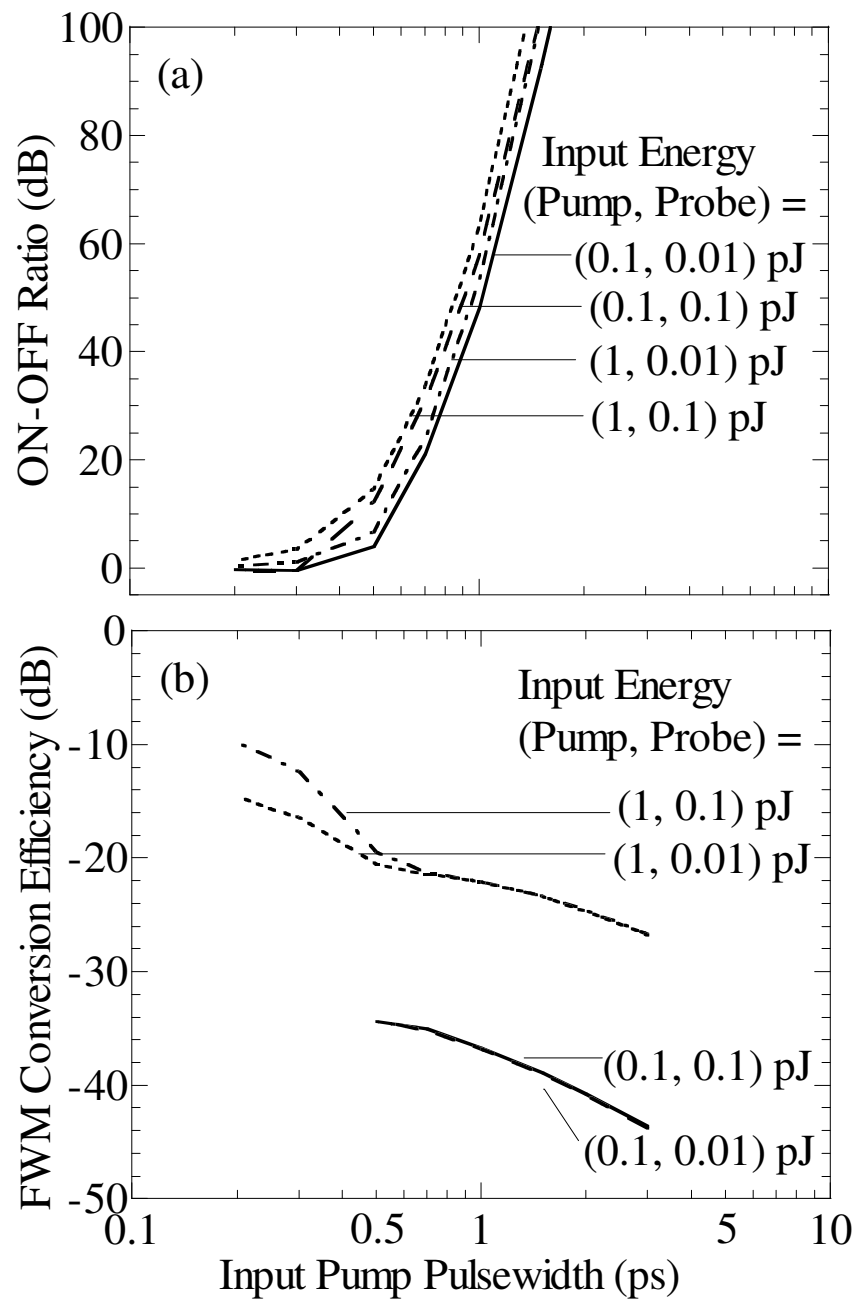

Fig. 8. (a) ON-OFF ratio and (b) FWM conversion efficiency. The input probe pulse energies are $0.01 \mathrm{pJ}$ and $0.1 \mathrm{pJ}$, and pulsewidth is $1 \mathrm{ps}$. The input pump pulse energies are $0.1 \mathrm{pJ}$ and $1 \mathrm{pJ}$. 


\section{Optical DEMUX characteristics in SOAs with multi-bit probe or pump pulses}

In this section, we will discuss the optical DEMUX characteristics in SOAs with multi-bit probe or multi-bit pump pulses and vice versa. The FWM signal generates only when the pump and probe pulses are injected simultaneously into SOAs. Therefore, all-optical demultiplexed signals can be extracted as the FWM signals from a time-multiplexed signal train as described in the Introduction. Here, the pump and probe pulses act as gating and gated pulses, respectively. For a solitary probe pulse, the overlap between the pump pulse and the FWM signal in the frequency domain decreases the ON-OFF ratio as described in the previous section. The overlap in the frequency domain increases with the decrease in the pump pulsewidth. Therefore, the ON-OFF ratio increases with the increase of pump pulsewidth. On the otherhand, for the multi-bit probe pulses, the overlap among the pulses in the time domain also decreases the ON-OFF ratio. This overlap mainly comes from the neighboring pulses in the time domain. To investigate the influence of the neighboring pulses, we simulate the optical DEMUX characteristics for a three-bit-stream of $250 \mathrm{Gbit} / \mathrm{s}$ in this section. We also evaluate the pattern effect on the DEMUX (based on FWM) signals caused by the probe pulses.

Figure 9 shows the schematic diagram for the simulation of the ON-OFF ratio of the alloptical DEMUX. The probe pulses are a three-bit-stream of $250 \mathrm{Gbit} / \mathrm{s}$. The peak position of the pump pulse is adjusted to that of the center pulse of the three probe pulses. Here, the ON-OFF ratio is defined as the ratio of the FWM signal energy obtained with the central input probe pulse of the three-bit-stream to the one obtained without the central input probe pulse. For the wider input pump pulse as indicated by the dashed lines, the FWM signal decreases and the ON-OFF ratio decreases, i.e., the crosstalk from the neighboring pulses increases.

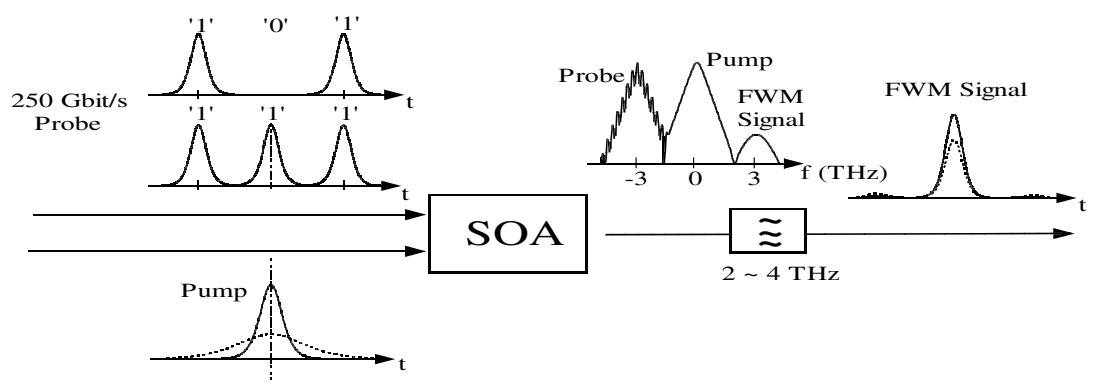

Fig. 9. A simple schematic diagram for the simulation of ON-OFF ratio of all-optical DEMUX. The input probe pulse repetition rate is $250 \mathrm{Gbit} / \mathrm{s}$ and pulsewidth is $1 \mathrm{ps}$. In the input probe pulse stream, ' $\mathbf{0}$ ' represents the signal is OFF and ' $\mathbf{1}$ ' represents the signal is ON.

Figure 10 shows the calculated ON-OFF ratio versus the input pump pulsewidth characteristics for the three-bit-stream. The input probe pulse energies are $0.1 \mathrm{pJ}$ and $0.01 \mathrm{pJ}$, and pulsewidth is $1 \mathrm{ps}$. The input pump energies are $0.01 \mathrm{pJ}, 0.1 \mathrm{pJ}$, and $1 \mathrm{pJ}$. For the wider input pump pulsewidth, the ON-OFF ratio decreases due to the overlap in the time domain among the pump and the neighboring of probe pulses. For an input pump pulsewidth of 3 ps, the ON-OFF ratio becomes about $20 \mathrm{~dB}$. This relatively large allowance in the pump 
pulsewidth is due to the fact that the pulsewidth of the probe pulses ( $1 \mathrm{ps})$ is set to be short compared with a bit interval of 4 ps $(250 \mathrm{Gbit} / \mathrm{s})$. On the other hand, with the decreasing in the pump pulsewidth, the ON-OFF ratio severely decreases due to the overlap in the frequency domain between the pump and the FWM signal pulse as explained in Fig. 8. This small allowance is attributed to the fact that the pump pulse energy is much stronger than that of the FWM signal. These results have an interesting information; the overlap in the frequency domain is more important than the overlap in the time domain for the design of the ultrafast all-optical DEMUX. As a result of the simulation, the optimum input pump pulsewidth range is $1 \mathrm{ps} \sim 3 \mathrm{ps}$ for an input probe pulsewidth of $1 \mathrm{ps}$.

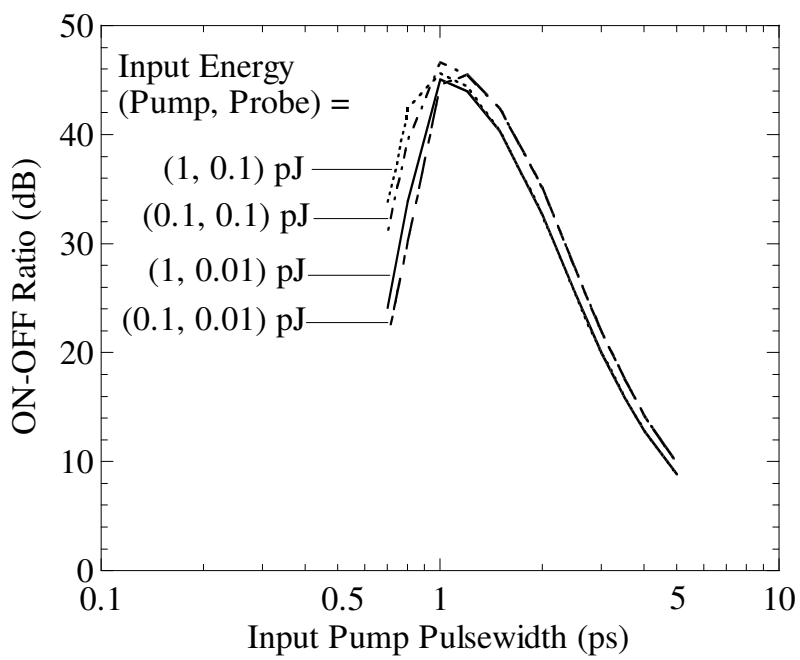

Fig. 10. ON-OFF ratio for a three-bit-stream probe. The input probe energies are $0.01 \mathrm{pJ}$ and $0.1 \mathrm{pJ}$, and pulsewidth is $1 \mathrm{ps}$. The input pump pulse energies are $0.1 \mathrm{pJ}$ and $1 \mathrm{pJ}$.

In the experiments reported so far, 100 - $6.3 \mathrm{Gbit} / \mathrm{s}$ (Kawanishi et al., 1994; Uchiyama et al., 1998), 200 - $6.3 \mathrm{Gbit} / \mathrm{s}$ (Morioka et al., 1996), 40 - $10 \mathrm{Gbit} / \mathrm{s}$ (Tomkos et al., 1999), and 100 $10 \mathrm{Gbit} / \mathrm{s}$ (Kirita et al., 1998) demultiplexing were performed. In our simulation/ modeling, we have considered the nonlinear effects, $\mathrm{CD}, \mathrm{CH}$ and $\mathrm{SHB}$ with the recovery times of 200 ps, $700 \mathrm{fs}$, and $60 \mathrm{fs}$, respectively (Hong et al., 1996). Because, we assumed a probe pulse repetition rate of $250 \mathrm{Gbit} / \mathrm{s}$, which is much faster than the recovery time of the $\mathrm{CD}$, the CD caused by the probe pulses remains when the following probe pulses are injected into the SOA. Therefore, the pattern effect may arise and deteriorate the DEMUX operation for the multi-bit probe pulses (Saleh \& Habbab, 1990). Here, we have considered the pattern effect of the probe bits because the different number of probe pulses is injected between the consecutive pump pulses depends on the bit pattern. Figure 11 shows the schematic diagram for the simulation of an optical DEMUX to investigate the pattern effect appearing at the DEMUX signals. The repetition rate and the pulsewidth of the input probe pulse are set to be $250 \mathrm{Gbit} / \mathrm{s}$ and $1 \mathrm{ps}$, respectively. We have simulated the FWM signals for the case that the different number of the probe pulses, n- 1 are injected before the demultiplexed signal is extracted. More number of probe pulses reduce the DEMUX (FWM) signals as shown by the dashed line (where, $\mathrm{n}=30$ ). 
$250 \mathrm{Gbit} / \mathrm{s}$ Probe
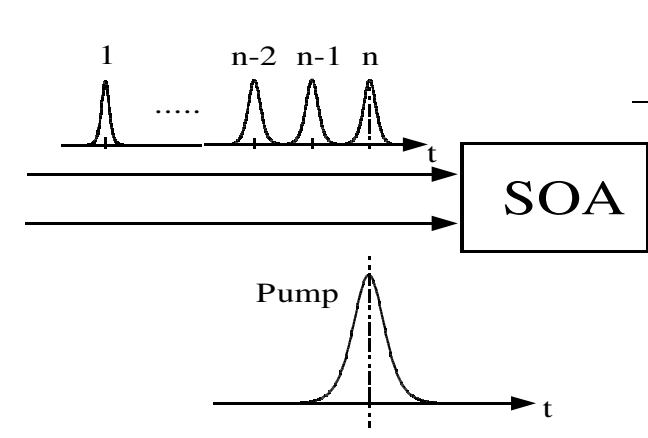

DEMUX Signal

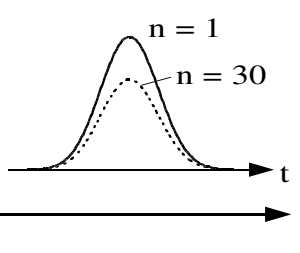

$2 \sim 4 \mathrm{THz}$

Fig. 11. Schematic diagram for the simulation of pattern effect on all-optical DEMUX operation. The input probe pulse repetition rate and pulsewidth are $250 \mathrm{Gbit} / \mathrm{s}$ and $1 \mathrm{ps}$, respectively. The different number of the probe pulses $n-1$ are injected before the demultiplexed signal is extracted.

Figure 12(a) shows an example of the pattern effect on the DEMUX signal waveforms. The input pump and probe pulse energies are $0.1 \mathrm{pJ}\left(\mathrm{E}_{\mathrm{p}}\right)$ and $0.1 \mathrm{pJ}\left(\mathrm{E}_{\mathrm{q}}\right)$, respectively. With increase the number of probe pulses, the FWM signal peak power decreases. The reduction in the peak power amounts to $7.4 \%$ for 30 probe pulses, while the waveforms remain unaffected. Fig. 12(b) shows the FWM signal energy versus the number of probe pulses. The closed circles show the calculated results and the solid line shows the fitted curve under the following approximation (Das, 2000; Das et al., 2001). The FWM signal is generated through the modulations in the refractive index and gain in the active region of SOAs. The modulation depths are proportional to both the carrier density and photon density because the modulation is created by the stimulated emission. Therefore, the FWM signal may also be proportional to the carrier density and photon density. The rate equation that describes the carrier density $N$ in the active region is given by

$$
\frac{d N}{d t}=\beta\left(N_{0}-N\right)-a P N
$$
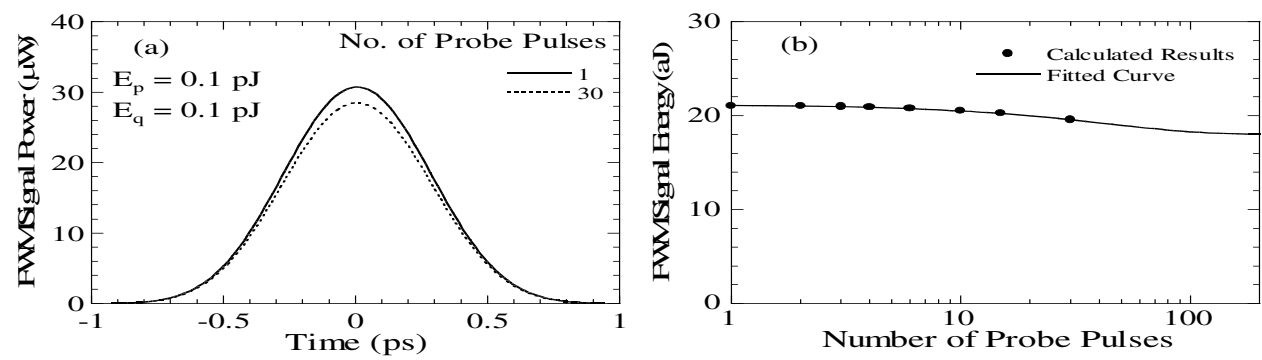

Fig. 12. (a) Pattern effect on the DEMUX signal waveforms and (b) FWM signal energy against number probe pulses. The input pump and probe energies are $0.1 \mathrm{pJ}$ and $0.1 \mathrm{pJ}$, respectively. 
where, $P$ is the photon density, $\beta\left(=1 / \tau_{\mathrm{s}}\right)$ is the recovery rate of the carrier density, $N_{0}$ is the carrier density at $t=0$ (before the injection of probe pulse), and $a$ is the coefficient of the stimulated emission. At $t=0, N=N_{0}$, then the solution of equation (17) is as follows:

$$
N=\frac{\beta N_{0}}{a P+\beta}\left[1+\frac{a P}{\beta} \exp \{-(a P+\beta) t\}\right]
$$

The repetition rate of the probe pulse is $250 \mathrm{GHz}$. Thus, we assume that the light with a constant photon density $P$ is injected. The duration of the probe bits is given by $t=n /\left(2.5 \times 10^{11}\right)$. Because, the FWM signal intensity is proportional to the carrier density $N$, the FWM signal intensity $S_{F W M}$ can be expressed as follows.

$$
S_{F W M}(t)=A[1+B \exp (-t / \tau)]
$$

where, $A=\beta N_{0} \tau, B=a P / \beta$ and $\tau=1 /(a P+\beta)$. In Fig. 12(b) the solid line shows the fitted curve using the equation (19) with the parameters, $A$ of 18.0 aJ, $B$ of 0.178 , and $\tau$ of 172 ps. Here, $A(1+B)$ corresponds to the maximum FWM signal intensity at $t=0, B$ is the constant representing the decrease in the FWM signal intensity caused by the probe pulses and $\tau$ is the effective recovery time of the carrier density depending on the input probe intensity. From equation (20), we obtained that the maximum fluctuation reaches to $\sim 15 \%$ for the infinite number of probe pulse train.

$$
\frac{S_{F W M}(0)-S_{F W M}(\infty)}{S_{F W M}(0)}=\frac{B}{1+B}
$$

Figure 13 shows another example of the pattern effects on the DEMUX signal for an input pump energy of $1 \mathrm{pJ}$ and a probe energy of $0.01 \mathrm{pJ}$. In this case, the FWM signal intensities are stronger than the results shown in Fig. 12, because the probe energy is 10 times lower and the pump energy is 10 times as stronger than in Fig. 12. The FWM signal energy decreases by only $0.03 \%$ for 30 probe pulses. We have obtained FWM signal energy $A$ of 62.0 aJ, $B$ of 0.012 , and $\tau$ of 200 ps from the fitted curve of Fig. 13(b). In this case, the FWM signal energy reduces only by $1.14 \%$ for the infinite number of probe pulses. We believe that such a small fluctuation is not an obstacle for the practical application. Although the results are not shown here, another set of the calculations are carried out shown in the Fig. 13, where the input pump and probe energy are $1 \mathrm{pJ}$ and $0.1 \mathrm{pJ}$, respectively. The input pump energy is 10 times stronger than that of Fig. 12. The FWM signal intensities were about 100 times stronger than the results shown in Fig. 12. The FWM signal peak power decreases by less than $3 \%$ for 30 probe pulses. From the fitting to the calculations, we have obtained that $A$ is $0.565 \mathrm{fJ}, B$ is 0.09 , and $\tau$ is 175 ps. Therefore, the FWM signal energy reduces by $9 \%$ for the infinite number of probe pulses in this condition. From these results, we can conclude that the intensity fluctuation of the FWM signal can be decreased by using the strong pump pulses or/and the weak input probe pulses.

The effective recovery time of the carrier density $\tau$ is defined as $\tau=1 /(a P+\beta)$. Therefore, in the weak limit of the probe pulses, $\tau$ should correspond to the carrier recovery time $\tau_{\mathrm{s}}$. In the weak probe case as shown in Fig. 13(b), $\tau$ is 200 ps and agrees with $\tau_{\mathrm{s}}$. In the case of the 
strong probe pulses, $\tau$ becomes short due to the stimulated emission caused by the probe pulses. For a strong probe pulse energy of $0.1 \mathrm{pJ}, \tau$ becomes smaller and they are $172 \mathrm{ps}$ and $175 \mathrm{ps}$ for pump pulse energies of $1 \mathrm{pJ}$ and $0.1 \mathrm{pJ}$, respectively. These results support our assumptions as mentioned above.
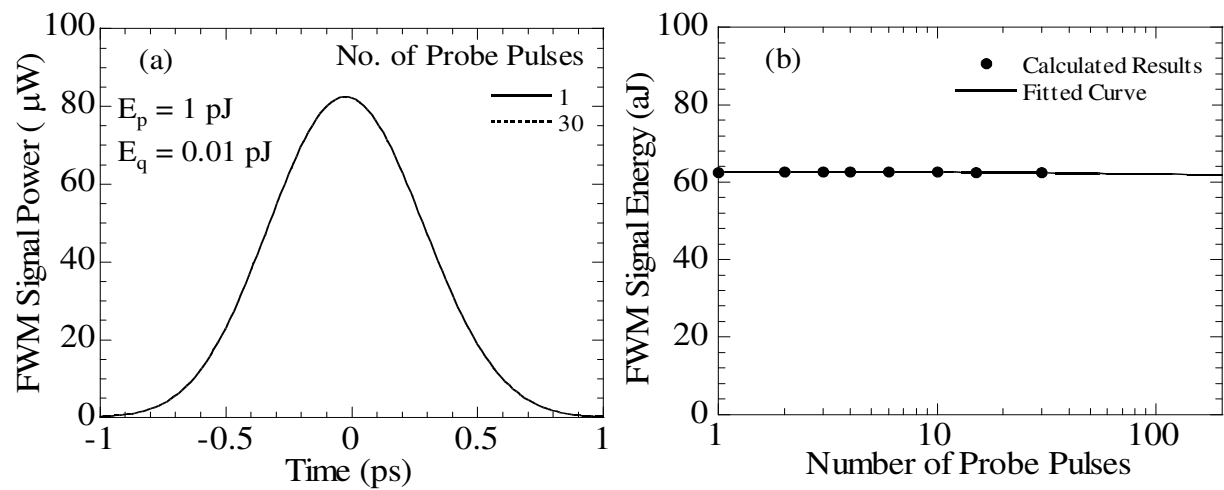

Fig. 13. (a) Pattern effect on the DEMUX signal waveforms and (b) FWM signal energy against number probe pulses. For this case, the input pump and probe energies are $1 \mathrm{pJ}$ and $0.01 \mathrm{pJ}$, respectively.

Figure 14 shows an example of temporal waveforms of demuliplexed signals from time multiplexed signals by repetitive pump pulses. The input pump and probe pulse energies are $1 \mathrm{pJ}$ and $0.01 \mathrm{pJ}$, respectively. The probe pulses are with a pulsewidth of $1 \mathrm{ps}$, sech ${ }^{2}$ shape and have a repetition rate of $250 \mathrm{GHz}$. The pump pulses are with a pulsewidth of $1 \mathrm{ps}, \mathrm{sech}^{2}$ shape and have a repetition rate of $62.5 \mathrm{GHz}$. Therefore, the $62.5 \mathrm{Gbit} / \mathrm{s}$ demultiplexed signals are selected once every four bits from the $250 \mathrm{Gbit} / \mathrm{s}$ signals. The FWM signal power is decreased by the strong input pump power due to the gain saturation and reaches to the constant value which is $\sim 23 \%$ of the FWM signal power among the solitary pulses. There will be no pattern effect due to the gain saturation caused by the pump power, because the pump pulses are injected continuously. In this particular case with a low probe pulse energy of $0.01 \mathrm{pJ}$, the pattern effect caused by the probe pulse is expected to be very small as shown in Fig. 13.

One of the most important effects we have not included in this modeling is an amplified spontaneous emission (ASE) noise which is generated in SOAs. However, a number of literature emphasized the importance of the ASE noise. Summerfield and Tucker (Summerfield \& Tucker, 1995) defined and measured the noise figure of an optical frequency converter based on FWM in an SOA. Diez et al., (Diez et al., 1997) defined the signal-to-background ratio (SBR) and investigated that different optimization criteria than for continuous waves apply as far as pulsed FWM applications concerned. Diez et al., (Diez et al., 1999) also reported a strong dependence of both conversion efficiency and SBR on pulsewidth and bit rate. This behavior has been attributed to the dynamics of the ASE, which is the main source of noise in an SOA.

Although the level of ASE strongly depends on the SOA structure and the operation conditions of SOAs, we have roughly compared with our calculated results and the ASE 
level on the assumption that the ASE level is $-40 \mathrm{dBm} / \mathrm{nm}$ (Diez et al., 1997). In Fig. 6(a), the FWM signal is directly compared with ASE level. The FWM signal is about $10 \mathrm{~dB}$ greater than the ASE level when the pump pulsewidth is 1 ps. In Fig. 6(b), the ASE level becomes $0.5 \mu \mathrm{W}$ if we use a filter with $2 \mathrm{THz}$ bandwidth. We can observe very clearly the FWM signal in the time domain. In Fig. 7, the ASE level becomes $\sim 8$ aJ if we use a filtering with $2 \mathrm{THz}$ bandwidth and select a time slot of 16 ps (i.e., consider a $62.5 \mathrm{GHz}$ repetition rate). Therefore, except for the conditions of a $0.1 \mathrm{pJ}$ pump pulse and a $0.01 \mathrm{pJ}$ probe pulse, the energy of FWM signal is greater than the ASE level. For more detailed comparison, it is recommended to take into account the ASE effect and its dynamic characteristics in the simulation.
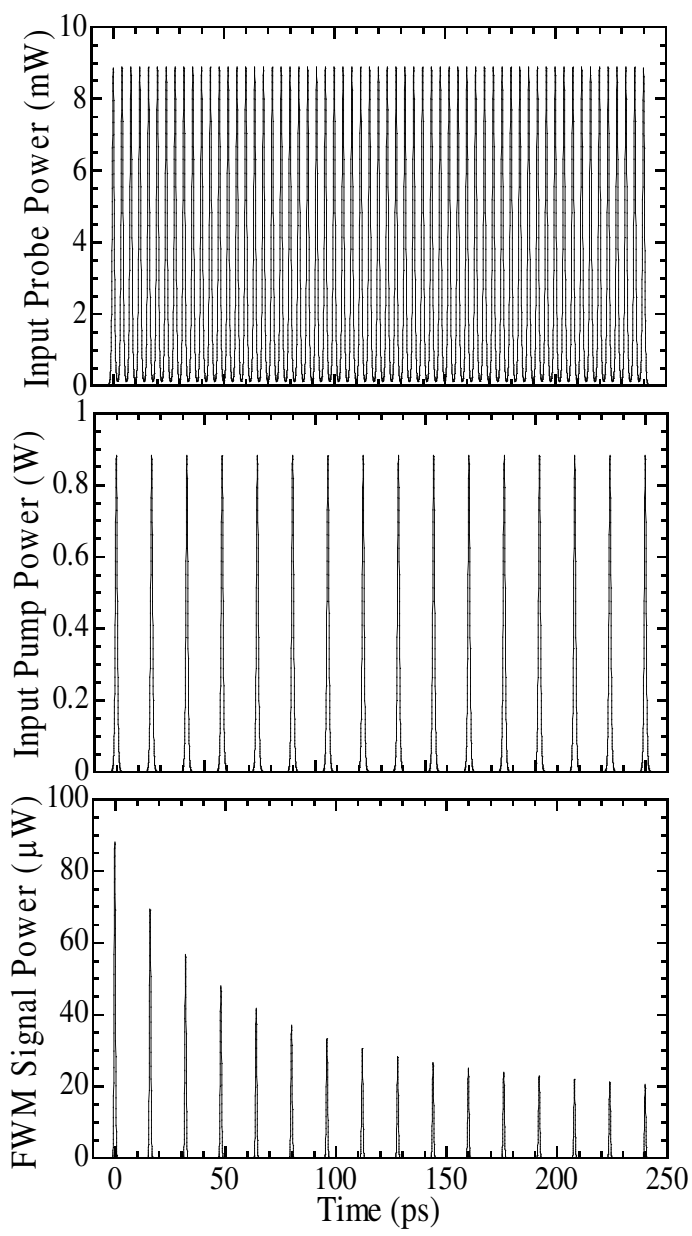

Fig. 14. DEMUX signal characteristics for the repetitive pump pulses. The input pump and probe pulse energies are $1 \mathrm{pJ}$ and $0.01 \mathrm{pJ}$, respectively. Here, top figure is the input probe pulses, middle figure is the input pump pulses and bottom figure is the generated FWM signal pulses. 


\section{Conclusion}

We have presented a detail analysis of all-optical DEMUX based on FWM in SOAs by solving the modified nonlinear Schrödinger equation using the FD-BPM. From this analysis, it was clarified that the optimization of the input pump pulsewidth is crucial to achieve a high ON-OFF ratio. We have obtained an optimum input pump pulsewidth of $1 \sim 3$ ps for 1 ps, 250 Gbit/s input probe pulses. The shorter limit of the pulsewidth is due to detuning between the pump and probe frequency, which is determined by the gain bandwidth of the SOA. In order to realize faster DEMUX operation, SOAs with broader gain bandwidth are required. We have also simulated pattern effects in the FWM signal. When the number of input probe pulses increases, the FWM signal power decreases, however; the FWM signal waveforms remain unaffected. The peak power fluctuation of the FWM signal can be reduced by using the strong pump pulses and/or weak probe pulses. The energy fluctuation of the FWM signal decreases to less than 1\% for a 30-bits, 250-Gbit/s input probe pulse train with a pulse energy of $0.01 \mathrm{pJ}$. This small fluctuation should not disturb the practical DEMUX operation. We also confirmed the DEMUX from time multiplexed signals by repetitive pump pulses. The strong energy pump pulses decrease the FWM signal intensity, however, there is no pattern effect due to gain saturation, because the pump pulses are injected continuously.

\section{Acknowledgments}

The authors would like to thank Dr. T. Kawazoe and Mr. Y. Yamayoshi for their helpful contribution to this work.

\section{References}

Agrawal, G. P. (1989). Nonlinear Fiber Optics. Academic Press, San Diego, Calif. ISBN 0-12045142-5.

Agrawal, G. P. \& Olsson, N. A. (1989). Self-phase modulation and spectral broadening of optical pulses in semiconductor laser and amplifiers. IEEE J. Quantum Electron., vol. 25, pp. 2297-2306, ISSN 0018-9197.

Aghajanpour, H.; Ahmadi, V. \& Razaghi, M. (2009). Ultra-short optical pulse shaping using semiconductor optical amplifier. Optics \& Laser Technology, vol. 41, pp. 654-658, ISSN 0030-3992.

Brigham, E. Oran (1988). The Fast Fourier Transform and Its Applications. Englewood Cliffs, N.J.: Prentice-Hall Inc. ISBN 0-13-307505-2.

Buxens, A.; Poulsen, H. N., Clausen, A. T., \& Jeppesen, P. (2000). All-optical OTDM-toWDM signal-format translation and OTDM add-drop functionality using bidirectional four wave mixing in semiconductor optical amplifier. Electron. Lett., vol. 36, pp. 156-158, ISSN 0013-5194.

Chung, Y. \& Dagli, N. (1990). An Assessment of finite difference beam propagation method. IEEE J. Quantum Electron., vol. 26, pp. 1335-39, ISSN 0018-9197.

Connelly, M. J.; Barry, L. P., Kennedy, B. F. \& Ried, D. A. (2008). Numerical analysis of fourwave mixing between picosecond mode-locked laser pulses in a tensile-strained bulk SOA. Optical and Quantum Electronics, vol. 40, pp. 411-418, ISSN 1572-817X. 
Conte, S. D. \& Boor, Carl de (1980). Elementary Numerical Analysis: An Algorithmic Approach, Third Edition, McGraw-Hill Book Company Co., Singapore. ISBN 0070124477.

Das, N. K. (2000). Numerical simulations of four-wave mixing among short optical pulses in semiconductor optical amplifiers by the beam propagation method. PhD dissertation, Yamagata University, Japan.

Das, N. K.; Yamayoshi, Y. \& Kawaguchi, H. (2000). Analysis of basic four-wave mixing characteristics in a semiconductor optical amplifier by beam propagation method. IEEE J. Quantum Electron., vol. 36, pp. 1184-1192, ISSN 0018-9197.

Das, N. K. \& Karmakar, N. C. (2008). Nonlinear propagation and wave mixing characteristics of pulses in semiconductor optical amplifiers. Microwave and Optical Technology Letters, vol. 50, pp. 1223-1227, ISSN 0895-2477.

Das, N. K.; Kawaguchi, H. \& Alameh, K. (2011). Advances in Optical Amplifiers, Paul Urquhart (Ed.), "Ch. 6: Impact of Pump-Probe Time Delay on the Four-Wave Mixing Conversion Efficiency in SOAs", InTech, Austria. ISBN 978-953-307-186-2.

Das, N. K.; Karmakar, N. C., Yamayoshi, Y. \& Kawaguchi, H. (2007). Four-wave mixing characteristics in SOAs with optimum time-delays between pump and probe pulses," Microwave and Optical Technology Letters, vol. 49, pp. 1182-1185, ISSN 08952477.

Das, N. K.; Yamayoshi, Y., Kawazoe, T. \& Kawaguchi, H. (2001). Analysis of optical DEMUX characteristics based on four-wave mixing in semiconductor optical amplifiers. IEEE /OSA J. Lightwave Technol., vol. 19, pp. 237-246, ISSN 0733-8724.

Das, N. K.; Kawazoe, T., Yamayoshi, Y. \& Kawaguchi, H. (2001). Analysis of optical phaseconjugate characteristics of picosecond four-wave mixing signals in semiconductor optical amplifiers. IEEE J. Quantum Electron., vol. 37, pp. 55-62, ISSN 0018-9197.

Das, N. K.; Karmakar, N. C., Yamayoshi, Y. \& Kawaguchi, H. (2005). Four-wave mixing characteristics among short optical pulses in semiconductor optical amplifiers with optimum time-delays, Proceedings of the 18th Annual Meeting of the IEEE Lasers and Electro-Optics Society 2005 (IEEE-LEOS2005), pp. 127-128, ISBN 0-7803-9217-5, Sydney, NSW, Australia, October 2005, IEEE Press (USA).

Dienes, A.; Heritage, J. P., Jasti, C. \& Hong, M. Y. (1996). Femtosecond optical pulse amplification in saturated media. J. Opt. Soc. Am. B, vol. 13, pp. 725-734, ISSN 07403224.

Diez, S.; Schmidt, C., Ludwig, R., Weber, H. G., Obermann, K., Kindt, S., Koltchanov, I. \& Petermann, K. (1997). Four-wave mixing in semiconductor optical amplifiers for frequency conversion and fast optical switching. IEEE J. Sel. Top. Quantum Electron., vol. 3, pp. 1131-1145, ISSN 1939-1404.

Diez, S.; Mecozzi, A. \& Mфrk, J. (1999). Bit rate and pulse width dependence of four-wave mixing of short pulses in semiconductor optical amplifiers. Opt. Lett., vol. 24, pp. 1675-1677, ISSN: 0146-9592.

Eiselt, M. (1995). Optimum pump pulse selection for demultiplexer application of four-wave mixing in semiconductor laser amplifiers. IEEE Photon. Technol. Lett., vol. 7, pp. 1312-1314, ISSN 1041-1135.

Gingrich, H. S.; Chumney, D. R., Sun, S.-Z., Hersee, S. D., Lester, L. F. \& Brueck, S. R. (1997). Broadly tunable external cavity laser diodes with staggered thickness multiple quantum wells. IEEE Photon. Technol. Lett., vol. 9, pp. 155-157, ISSN 1041-1135. 
Hong, M. Y.; Chang, Y. H., Dienes, A., Heritage, J. P., Delfyett, P. J., Dijaili, Sol \& Patterson, F. G. (1996). Femtosecond self- and cross-phase modulation in semiconductor laser amplifiers. IEEE J. Sel. Top Quantum Electron., vol. 2, pp. 523-539, ISSN 1939-1404.

Inoue, J. \& Kawaguchi, H. (1998). Highly nondegenerate four-wave mixing among subpicosecond optical pulses in a semiconductor optical amplifier," IEEE Photon. Technol. Lett., vol. 10, pp. 349-351, ISSN 1041-1135.

Kikuchi, K. \& Matsumura, K. (1998). Transmission of 2-ps optical pulses at $1550 \mathrm{~nm}$ over 40$\mathrm{km}$ standard fiber using midspan optical phase conjugation in semiconductor optical amplifiers. IEEE Photon. Technol. Lett., vol. 10, pp. 1410-1412, ISSN 10411135.

Kawanishi, S.; Okamoto, K., Ishii, M., Kamatani, T., Takara, H., \& Uchiyama, K. (1997). Alloptical time-division-multiplexing on four-wave mixing in a travelling-wave semiconductor laser amplifier. Electron. Lett., vol. 33, pp. 976-977, ISSN 0013-5194.

Kawanishi, S.; Morioka, T., Kamatani, O., Takara, H., Jacob, J.M., \& Saruwatari, M. (1994). $100 \mathrm{Gbit} / \mathrm{s}$ all-optical demultiplexing using four-wave mixing in a travelling wave laser diode amplifier. Electron. Lett., vol. 30, pp. 981-982, ISSN 0013-5194.

Kirita, H.; Hashimoto, Y., \& Yokoyama, H. (1998). All-optical signal processing at over 100 Gbit/s with nonlinear effects in semiconductor lasers. Tech. Dig. Int'l. Trop. Workshop on Contemporary Technologies (CPT '98), Paper Pc-14, Tokyo, Japan.

Koltchanov, I.; Kindt, S., Petermann, K., Diez, S., Ludwig, R., Schnabel, R., \& Weber, H. G. (1996). Gain dispersion and saturation effects in four-wave mixing in semiconductor laser amplifiers. IEEE J. Quantum Electron., vol. 32, pp. 712-720, ISSN 0018-9197.

Leuthold, J.; Mayer, M., Eckner, J., Guekos, G., Melchior, H. and Zellweger, Ch. (2000). Material gain of bulk $1.55 \mu \mathrm{m}$ InGaAsP/InP semiconductor optical amplifiers approximated by a polynomial model. J. Appl. Phys., vol. 87, pp. 618-620, ISSN.

Mecozzi, A. \& M $\phi r k$, J. (1997). Saturation effects in nondegenerate four-wave mixing between short optical pulses in semiconductor laser amplifiers. IEEE J. Sel. Top Quantum Electron., vol. 3, pp. 1190-1207, ISSN 1939-1404.

Mecozzi, A.; D’Ottavi, A., Iannone, E., \& Spano, P. (1995). Four-wave mixing in travellingwave semiconductor amplifiers. IEEE J. Quantum Electron., vol. 31, pp. 689-699, ISSN 0018-9197.

Mikami, O.; Noguchi, Y., Yasaka, H., Magari, K. \& Kondo, S. (1991). Emission spectral width broadening for InGaAsP/InP superluminescent diodes. IEE Proc. J Optoelectron., vol. 138, pp. 133-137, ISSN: 0267-3932.

Mprk, J. \& Mecozzi, A. (1997). Theory of nondegenerate four-wave mixing between pulses in a semiconductor waveguide. IEEE J. Quantum Electron., vol. 33, pp. 545-555, ISSN 0018-9197.

Morioka, T.; Takara, H., Kawanishi, S., Uchiyama, K., \& Saruwatari, M. (1996). Polarisationindependent demultiplexing up to $200 \mathrm{~Gb} / \mathrm{s}$ using four-wave mixing in a semiconductor laser amplifier. Electron. Lett., vol. 32, pp. 840-841, ISSN 0013-5194.

Okamoto, K. (1992). Theory of Optical Waveguides, Corona Publishing Co., Tokyo; Ch. 7 (in Japanese). ISBN 4-339-00602-5.

Razaghi, M.; Ahmadi, A., Connelly, M. J. \& Madanifar, K. A. (2009a). Numerical modelling of sub-picosecond counter propagating pulses in semiconductor optical amplifiers. Proceedings of the 9th International Conference on Numerical Simulation of Optoelectronic 
Devices 2009 (NUSOD' 09), pp. 59-60, ISBN 978-1-4244-4180-8, GIST, Gwangju, South Korea, September 2009, IEEE Press (USA).

Razaghi, M.; Ahmadi, A., \& Connelly, M. J. (2009b). Comprehensive finite-difference timedependent beam propagation model of counter propagating picosecond pulses in a semiconductor optical amplifier. IEEE/OSA J. Lightwave Technol., vol. 27, pp. 31623174, ISSN 0733-8724.

Saleh, A. A. A. \& Habbab, I. M. I. (1990). Effects of semiconductor-optical-amplifier nonlinearity on the performance of high-speed intensity-modulation lightwave systems. IEEE Trans. Commun., vol. 38, pp. 839-846, ISSN 0090-6778.

Seki, K.; Kamiya, T. and Yanai, H. (1981). Effect of waveguiding properties on the axial mode competition in stripe-geometry semiconductor lasers. IEEE J. Quantum Electron., vol. 17, pp. 706-713, ISSN 0018-9197.

Shtaif, M. \& Eisenstein, G. (1995). Analytical solution of wave mixing between short optical pulses in semiconductor optical amplifier. Appl Phys. Lett. 66, pp. 1458-1460, ISSN 0003-6951.

Shtaif, M.; Nagar, R. \& Eisenstein, G. (1995). Four-wave mixing among short optical pulses in semiconductor optical amplifiers. IEEE Photon Technol. Lett. 7, pp. 1001-1003. ISSN 1041-1135.

Shtaif, M. \& Eisenstein, G. (1996). Calculation of bit error rates in all-optical signal processing applications exploiting nondegenerate four-wave mixing in semiconductor optical amplifiers. IEEE/OSA J. Lightwave. Technol., vol. 14, pp. 20692077, ISSN 0733-8724.

Summerfield, M. A. \& Tucker, S. R. (1995). Noise figure and conversion efficiency of fourwave mixing in semiconductor optical amplifiers. Electron. Lett., vol. 31, pp. 11591160, ISSN 0013-5194.

Sauter, E. G. (1996). Nonlinear Optics. John Wiley \& Sons, Inc. New York. ISBN 0-471-148601.

Tang, J. M. \& Shore, K. A. (1998). Influence of probe depletion and cross-gain modulation on four-wave mixing of picosecond optical pulses in semiconductor optical amplifiers," IEEE Photon. Technol. Lett., vol. 10, pp. 1563-1565, ISSN 1041-1135.

Tang, J. M. \& Shore, K. A. (1999). Active picosecond optical phase compression in semiconductor optical amplifiers. IEEE J. Quantum. Electron., vol. 35, pp. 93-100, ISSN 0018-9197.

Tang, J. M. \& Shore, K. A. (1999). Characteristics of optical phase conjugation of picosecond pulses in semiconductor optical amplifiers. IEEE J. Quantum. Electron., vol. 35, pp. 1032-1040, ISSN 0018-9197.

Tang, J. M.; Spencer, P. S. \& Shore, K. A. (1998). The influence of gain compression on picosecond optical pulses in semiconductor optical amplifiers. J. Mod. Opt., vol. 45, pp. 1211-1218, ISSN: 0950-0340.

Tomkos, I.; Zacharopoulos, I., Syvridis, D., Calvani, R., Cisternino, F., \& Riccardi, E. (1999). All-optical demultiplexing/shifting of 40-Gb/s OTDM optical signal using dualpump wave mixing in bulk semiconductor optical amplifier. IEEE Photon. Technol. Lett., vol. 11, pp. 1464-1466, ISSN 1041-1135.

Uchiyama, K.; Kawanishi, S., \& Saruwatari, M. (1998). 100-Gb/s multiple-channel output optical OTDM demultiplexing using multichannel four-wave mixing in a 
semiconductor optical amplifier. IEEE Photon. Technol. Lett., vol. 10, pp. 890-892, ISSN 1041-1135.

Vahala, K. J.; Zhou, J., Geraghty, D., Lee, R., Newkirk, M., \& Miller, B. (1996). Four-wave mixing in semiconductor travelling-wave amplifiers for wavelength conversion in all-optical networks, in T. P. Lee ed. Current Trends in Optical Amplifiers and Their Applications. World Scientific, Singapore.

Xie, C.; Ye, P. \& Lin, J. (1999). Four-wave mixing between short optical pulses in semiconductor optical amplifiers with the consideration of fast gain saturation. IEEE Photon. Technol. Lett., vol. 11, pp. 560-562, ISSN 1041-1135.

Yariv, A. (1991). Optical Electronics, 4th Edition, Saunders College Publishing, San Diego. ISBN 0-03-053239-6. 


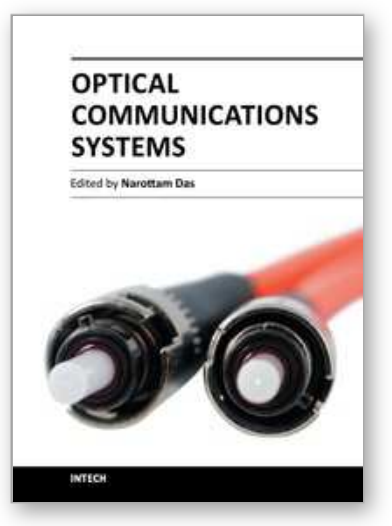

\author{
Optical Communications Systems
}

Edited by Dr. Narottam Das

ISBN 978-953-51-0170-3

Hard cover, 262 pages

Publisher InTech

Published online 07, March, 2012

Published in print edition March, 2012

Optical communications systems are very important for all types of telecommunications and networks. They consists of a transmitter that encodes a message into an optical signal, a channel that carries the signal to its destination, and a receiver that reproduces the message from the received optical signal. This book presents up to date results on communication systems, along with the explanations of their relevance, from leading researchers in this field. Its chapters cover general concepts of optical and wireless optical communication systems, optical amplifiers and networks, optical multiplexing and demultiplexing for optical communication systems, and network traffic engineering. Recently, wavelength conversion and other enhanced signal processing functions are also considered in depth for optical communications systems. The researcher has also concentrated on wavelength conversion, switching, demultiplexing in the time domain and other enhanced functions for optical communications systems. This book is targeted at research, development and design engineers from the teams in manufacturing industry; academia and telecommunications service operators/ providers.

\title{
How to reference
}

In order to correctly reference this scholarly work, feel free to copy and paste the following:

Narottam Das and Hitoshi Kawaguchi (2012). Optical Demultiplexing Based on Four-Wave Mixing in Semiconductor Optical Amplifiers, Optical Communications Systems, Dr. Narottam Das (Ed.), ISBN: 978-95351-0170-3, InTech, Available from: http://www.intechopen.com/books/optical-communications-systems/opticaldemultiplexing-based-on-four-wave-mixing-in-semiconductor-optical-amplifiers-

\section{INTECH}

open science | open minds

\section{InTech Europe}

University Campus STeP Ri

Slavka Krautzeka 83/A

51000 Rijeka, Croatia

Phone: +385 (51) 770447

Fax: +385 (51) 686166

www.intechopen.com

\section{InTech China}

Unit 405, Office Block, Hotel Equatorial Shanghai

No.65, Yan An Road (West), Shanghai, 200040, China 中国上海市延安西路65号上海国际贵都大饭店办公楼 405 单元

Phone: +86-21-62489820

Fax: $+86-21-62489821$ 
(C) 2012 The Author(s). Licensee IntechOpen. This is an open access article distributed under the terms of the Creative Commons Attribution 3.0 License, which permits unrestricted use, distribution, and reproduction in any medium, provided the original work is properly cited. 